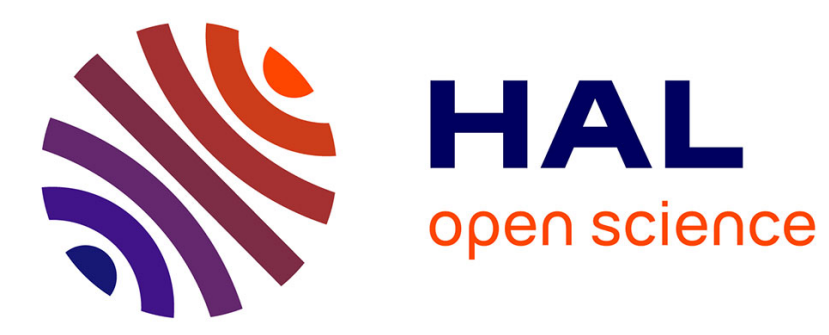

\title{
Derivation of a poroelastic elliptic membrane shell model
}

Andro Mikelic, Josip Tambača

\section{To cite this version:}

Andro Mikelic, Josip Tambača. Derivation of a poroelastic elliptic membrane shell model. Applicable Analysis, 2019, 98 (1-2), pp.136-161. 10.1080/00036811.2018.1430784 . hal-01488396

\section{HAL Id: hal-01488396 https://hal.science/hal-01488396}

Submitted on 13 Mar 2017

HAL is a multi-disciplinary open access archive for the deposit and dissemination of scientific research documents, whether they are published or not. The documents may come from teaching and research institutions in France or abroad, or from public or private research centers.
L'archive ouverte pluridisciplinaire HAL, est destinée au dépôt et à la diffusion de documents scientifiques de niveau recherche, publiés ou non, émanant des établissements d'enseignement et de recherche français ou étrangers, des laboratoires publics ou privés. 


\title{
Derivation of a poroelastic elliptic membrane shell model
}

\author{
Andro Mikelić ${ }^{*}$ \\ Univ Lyon, Université Claude Bernard Lyon 1, CNRS UMR 5208, \\ Institut Camille Jordan, 43 blvd. du 11 novembre 1918, \\ F-69622 Villeurbanne cedex, France \\ tel: +33 (0) 426234548; e-mail: mikelic@univ-lyon1.fr \\ Josip Tambača \\ Department of Mathematics \\ University of Zagreb \\ Bijenička 30, 10000 Zagreb, Croatia \\ tel: +385 (0)1 4605790; e-mail: tambaca@math.hr
}

February 19, 2017

\begin{abstract}
A derivation of the model for a poroelastic elliptic membrane shell is undertaken. The flow and deformation in a three-dimensional shell domain is described by the quasi-static Biot equations of linear poroelasticity. We consider the limit when the shell thickness goes to zero and look for the limit equations. Using the technique developed in the seminal articles by Ciarlet, Lods, Miara et al and the recent results on the rigorous derivation of the equations for poroelastic plates and flexural poroelastic shells by Marciniak-Czochra, Mikelić and Tambača, we present a rigorous derivation of the linear poroelastic elliptic membrane shell model. After rescaling, the corresponding velocity and the pressure field are close in the $C\left([0, T] ;\left(H_{x}^{1}\right)^{2} \times\left(L_{x}^{2}\right)^{2}\right)$ norm and the stresses in $C\left([0, T] ;\left(L_{x}^{2}\right)^{9}\right)$ norm. In the case of a spherical membrane shell we confirm the results by Taber from the literature.
\end{abstract}

Keywords: Membrane poroelastic shell, Biot's quasi-static equations, elliptic-parabolic systems, asymptotic methods

AMS subject classification: $\quad 35 \mathrm{~B} 25,74 \mathrm{~F} 10,74 \mathrm{~K} 25,74 \mathrm{Q} 15$, MSC $76 \mathrm{~S}$

\section{Contents}

1 Introduction

2 Geometry of Shells and Setting of the Problem 4

3 Problem setting in curvilinear coordinates and the main results $\quad 7$

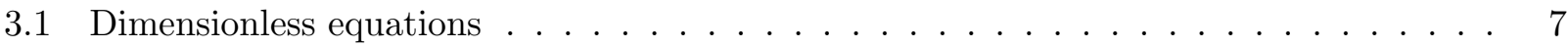

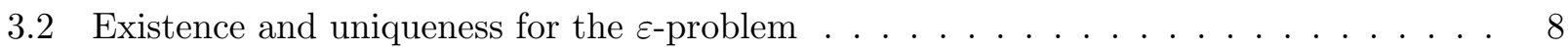

3.3 Problem in Curvilinear Coordinates and the Scaled Problem . . . . . . . . . . . . . 8

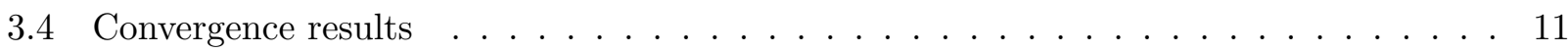

4 A priori estimates

\footnotetext{
${ }^{*}$ Corresponding author
} 


\section{Introduction}

The present work is devoted to the derivation of a model of the poroelastic elliptic membrane shell.

We follow the standpoint of Ciarlet et al, who derived the Kirchhoff-Love models for thin elastic bodies in the zero thickness limit (see $[6,7,8]$ ).

While this approach to the effective behavior of three-dimensional linearized elastic bodies is well-established, much less attention has been paid to the poroelastic thin bodies. As the recent example of the special issue of the journal Transport in Porous Media [34, 19] shows, this is likely to change. Also there is a related experimental work, see [17].

The poroelastic bodies are characterized by the simultaneous presence of the deformation and the filtration (flow). They are described by the quasi-static Biot's system of PDE's. It couples the Navier equations of linearized elasticity, containing the pressure gradient, with the mass conservation equation involving the fluid content change and divergence of the filtration velocity. The filtration velocity is the relative velocity for the upscaled fluid-structure problem and obeys Darcy's law. The fluid content change is proportional to the pressure and the elastic body compression. In the quasistatic Biot's system the mechanical part is elliptic in the displacement and the flow equation has a parabolic operator for the pressure. For more modeling details we refer to [10], [21] and [33] and for the mathematical theory to [28], [26] and [23].

The simplest relevant two dimensional poroelastic thin body is a poroelastic plate. A physically relevant choice of the time scale and the related coefficient size was set up by Marciniak-Czochra and Mikelić in [20]. They rigorously derived the effective equations for the Kirchhoff-Love-Biot poroelastic plate in the zero thickness limit of the 3D quasi-static Biot equations. The limiting zero thickness procedure is seriously affected by the presence of coupling structure-flow. As in the purely elastic case, the specificity of the poroelastic plate model from [20] is that the limit model contains simultaneously both flexural and membrane equations. This remarkable property does not transfer to the shells.

Following both Ciarlet et al zero thickness limit approach to flexural linearized shells and handling of the Biot quasi-static equations in a thin domain by Marciniak-Czochra and Mikelić, Mikelić and Tambača have undertaken the derivation of the equation for a linear flexural poroelastic shell in [22]. In this article we undertake derivation of a model for linear elliptic membrane poroelastic shell through the same type of the limit procedure.

The coupling elastic structure - flow is scaled as in [20]. It corresponds to the physical parameters leading to the quasi-static diphasic Biot's equations for the displacement and the pressure. As in [30] and [31], it means that the characteristic time scale is of Taber and Terzaghi and in the dimensionless form there will be the ratio between the width and length squared, multiplying Laplacean of the pressure. The flexural and the membrane poroelastic shells correspond to different regimes of the filtration, different sizes of the applied contact forces, different geometries of the shell and different boundary conditions. In our case they are applied at the top and the bottom boundaries. For the membrane shell case, we impose a given inflow/outflow velocity of order of the characteristic filtration velocity through a shell of width $\ell$. The applied contact forces at the same top/bottom boundaries should be of order of the pressure drop between these boundaries. We recall that in the case of the flexural poroelastic shell, studied in [22], the contact forces at the top/bottom boundaries are an order of magnitude smaller and even smaller than the related inflow/outflow velocities. 
The motivation for studying the flexural poroelastic shells comes from the industrial filters modeling. For instance, the results from [20] and [22] can be applied to the modeling of the air filters for the cars, while some oil car filters can be modeled as membrane poroelastic shells.

The motivation for studying the membrane poroelastic shells also comes from the biomechanics. An important example is the study of the mechanical behavior of fluid-saturated large living bone tissues. We recall that the bulk modulus of the bone is much larger than the bulk moduli of the soft tissues and the bone deformation is small. A full physiological understanding of the bone modeling would provide insight to important clinical problems which concern bones. For detailed review we refer to [27] and [11]. Many other living structures are fluid-saturated membrane shells, see e.g. [13].

Another modeling question, raised in [11], is of the modeling of the elastic wave propagation in a bone. As the Biot theory was originally developed to describe the wave propagation, the subject attracted attention. The reader can consult [3], [15] and [32] and references therein. With our scaling, our spatial operators do not coincide with models from these references, but rather with Taber's works [30] and [31]. Furthermore, the dynamic models of the diphasic Biot equations for a viscous fluid exhibit memory effects, as proposed by Biot through the introduction of the viscodynamic operator (see [33]). The homogenization derivation of the dynamic diphasic Biot's equations gives the memory terms (see [9]) for a viscous fluid. If the pores are filled by an ideal fluid, there are no memory effects (see [16]). The analysis of the relationship between the dynamic and the quasi-static diphasic Biot equation was undertaken in [24] and there are scalings when the memory effects are not important. But in general it is not possible just to add the acceleration to the quasi-static Biot system. Hence modeling of the elastic waves propagation in poroelastic plates/shell requires some future research.

Since in [23], the quasi-static Biot equations are obtained by homogenization of a pore scale fluidstructure problem, one can raise question why we do not study simultaneously homogenization of the fluid-structure problem and the zero thickness limit. In the applications we have in mind (industrial filters, living tissues...) the thickness is much bigger than the RVE size and such approach does not make much sense. For some other problems like the study of the overall behavior of curved layers of living cells, having a thickness of one cell, the simultaneous homogenization and singular perturbation would give new models.

The flexural shell model is formulated on a subspace of infinitesimally inextensional displacements involving boundary conditions, usually denoted by $V_{F}$. However this function space for some geometries and boundary conditions turns to be trivial. In this case a model for extensional displacements is necessary. In this paper we focus on the shells with elliptic surfaces which are clamped at the whole boundary. The model in this case is called elliptic membrane shell model. In the case of the classical elasticity the membrane effects are measured by the change of the metric of the shell. This is different with the case of the flexural shell model where the potential energy is measured by the change in the curvature tensor. This difference results in a simpler model for the membrane case and lower order derivatives involved in the formulation. Remaining cases in which $V_{F}=\{0\}$ as well are covered by the generalized membrane shell model (an example is a tube clamped at ends). However, the formulation is given in abstract spaces, see [5].

Derivation of the present model is more difficult than the derivation of the classical elliptic membrane shell model starting from the three-dimensional linearized [7]. Namely, we are dealing with an additional equation for the additional unknown (pressure). We use the results derived in the classical static case, see [5], as much as possible, but Biot's equations are quasi-static and, therefore, time dependent. Presence of the additional independent variable (time) requires special attention and careful analysis. Note also that in some parts this derivation is more demanding than the derivation of the poroelastic flexural shell model from [22] since here we have weaker a priori estimates in strains and we have to obtain the same convergences of the tangential displacements as in the flexural case. Finally, we recall that the flexural shell models are characterized by the presence of the 4th order differential operators and for the membrane shell models the differential operators are of the 2nd order. 


\section{Geometry of Shells and Setting of the Problem}

We are starting by recalling the basic facts of geometry of shells. The text follows [22], but it is shorter since some terms are not needed in the membrane model. Namely, lower order derivatives are sufficient to express the membrane effects.

Throughout this paper we use boldfaced letters for vectors or matrices. The only exceptions are points in the Euclidean spaces (e.g., $x, y$, ). $\mathbb{R}^{n \times m}$ denotes the space of all $n$ by $m$ matrices and the subscript sym denotes its subspace of symmetric matrices. By $L^{2}$ we denote the Lebesgue space of the square integrable functions, while $H^{1}$ stands for the Sobolev space.

Let the surface $\mathcal{S}$ is given as $\mathcal{S}=\mathbf{X}\left(\bar{\omega}_{L}\right)$, where $\omega \subset \mathbb{R}^{2}$ be an open bounded and simply connected set with Lipschitz-continuous boundary $\partial \omega_{L}$ and $\mathbf{X}: \bar{\omega}_{L} \rightarrow \mathbb{R}^{3}$ is a smooth injective immersion (that is $\mathbf{X} \in C^{3}$ and $3 \times 2$ matrix $\nabla \mathbf{X}$ is of rank two). Thus the vectors $\mathbf{a}_{\alpha}(y)=\partial_{\alpha} \mathbf{X}(y), \alpha=1,2$, are linearly independent for all $y \in \bar{\omega}_{L}$ and form the covariant basis of the tangent plane to the 2 -surface $\mathcal{S}$. Let $\Omega_{L}^{\ell}=\omega_{L} \times(-\ell / 2, \ell / 2)$. In this paper we study the deformation and the flow in a three-dimensional poroelastic shell $\tilde{\Omega}_{L}^{\ell}=\mathbf{r}\left(\Omega_{L}^{\ell}\right), L, \ell>0$, where the injective mapping $\mathbf{r}$ is given by

$$
\mathbf{r}=\mathbf{r}\left(y, x_{3}\right)=\mathbf{X}(y)+x_{3} \mathbf{a}_{3}(y), \quad \mathbf{a}_{3}(y)=\frac{\mathbf{a}_{1}(y) \times \mathbf{a}_{2}(y)}{\left|\mathbf{a}_{1}(y) \times \mathbf{a}_{2}(y)\right|},
$$

for $x_{3} \in(-\ell / 2, \ell / 2)$ and $\left(y_{1}, y_{2}\right) \in \omega_{L}$, diam $\left(\omega_{L}\right)=L$. The contravariant basis of the plane spanned by $\mathbf{a}_{1}(y), \mathbf{a}_{2}(y)$ is given by the vectors $\mathbf{a}^{\alpha}(y)$ defined by $\mathbf{a}^{\alpha}(y) \cdot \mathbf{a}_{\beta}(y)=\delta_{\beta}^{\alpha}$. We extend these bases to the basis of the whole space $\mathbb{R}^{3}$ by the vector $\mathbf{a}_{3}(y)$ given in $(2.1)\left(\mathbf{a}^{3}(y)=\mathbf{a}_{3}(y)\right)$. Now we collect the local contravariant and covariant bases into the matrix functions

$$
\mathbf{Q}=\left[\begin{array}{lll}
\mathbf{a}^{1} & \mathbf{a}^{2} & \mathbf{a}^{3}
\end{array}\right], \quad \mathbf{Q}^{-1}=\left[\begin{array}{c}
\mathbf{a}_{1}^{T} \\
\mathbf{a}_{2}^{T} \\
\mathbf{a}_{3}^{T}
\end{array}\right]
$$

The first fundamental form of the surface $\mathcal{S}$, or the metric tensor, in covariant $\mathbf{A}_{c}=\left(a_{\alpha \beta}\right)$ or contravariant $\mathbf{A}^{c}=\left(a^{\alpha \beta}\right)$ components are given respectively by

$$
a_{\alpha \beta}=\mathbf{a}_{\alpha} \cdot \mathbf{a}_{\beta}, \quad a^{\alpha \beta}=\mathbf{a}^{\alpha} \cdot \mathbf{a}^{\beta}, \quad \alpha, \beta=1,2 .
$$

Note here that because of continuity of $\mathbf{A}^{c}$ and compactness of $\bar{\omega}_{L}$, there are constants $M^{c} \geq m^{c}>0$ such that

$$
m^{c} \mathbf{x} \cdot \mathbf{x} \leq \mathbf{A}^{c}(y) \mathbf{x} \cdot \mathbf{x} \leq M^{c} \mathbf{x} \cdot \mathbf{x}, \quad \mathbf{x} \in \mathbb{R}^{3}, y \in \bar{\omega}_{L} .
$$

These estimates, with different constants, hold for $\mathbf{A}_{c}$ as well, as it is the inverse of $\mathbf{A}^{c}$. The second fundamental form of the surface $\mathcal{S}$, also known as the curvature tensor, in covariant $\mathbf{B}_{c}=\left(b_{\alpha \beta}\right)$ or mixed components $\mathbf{B}=\left(b_{\alpha}^{\beta}\right)$ are given respectively by

$$
b_{\alpha \beta}=\mathbf{a}^{3} \cdot \partial_{\beta} \mathbf{a}_{\alpha}=-\partial_{\beta} \mathbf{a}^{3} \cdot \mathbf{a}_{\alpha}, \quad b_{\alpha}^{\beta}=\sum_{\kappa=1}^{2} a^{\beta \kappa} b_{\kappa \alpha}, \quad \alpha, \beta=1,2 .
$$

The Christoffel symbols $\boldsymbol{\Gamma}^{\kappa}$ are defined by

$$
\Gamma_{\alpha \beta}^{\kappa}=\mathbf{a}^{\kappa} \cdot \partial_{\beta} \mathbf{a}_{\alpha}=-\partial_{\beta} \mathbf{a}^{\kappa} \cdot \mathbf{a}_{\alpha}, \quad \alpha, \beta, \kappa=1,2 .
$$

We will sometime use $\Gamma_{\alpha \beta}^{3}$ for $b_{\alpha \beta}$. The area element along $\mathcal{S}$ is $\sqrt{a} d y$, where $a:=\operatorname{det} \mathbf{A}_{c}$. By $(2.3)$ it is uniformly positive, i.e., there is $m_{a}>0$ such that

$$
0<m_{a} \leq a(y), \quad y \in \bar{\omega}_{L} .
$$


In order to describe our results we also need the following differential operators:

$$
\begin{aligned}
\gamma_{\alpha \beta}(\mathbf{v}) & =\frac{1}{2}\left(\partial_{\alpha} v_{\beta}+\partial_{\beta} v_{\alpha}\right)-\sum_{\kappa=1}^{2} \Gamma_{\alpha \beta}^{\kappa} v_{\kappa}-b_{\alpha \beta} v_{3}, \quad \alpha, \beta=1,2, \\
\left.n_{\alpha \beta}\right|_{\beta} & =\partial_{\beta} n_{\alpha \beta}+\sum_{\kappa=1}^{2} \Gamma_{\beta \kappa}^{\alpha} n_{\beta \kappa}+\sum_{\kappa=1}^{2} \Gamma_{\beta \kappa}^{\beta} n_{\alpha \kappa}, \quad \alpha, \beta=1,2,
\end{aligned}
$$

defined for smooth vector fields $\mathbf{v}$ and tensor fields $\mathbf{n}$.

The upper face (respectively lower face) of the shell $\tilde{\Omega}_{L}^{\ell}$ is $\tilde{\Sigma}_{L}^{\ell}=\mathbf{r}\left(\omega_{L} \times\left\{x_{3}=\ell / 2\right\}\right)=\mathbf{r}\left(\Sigma_{L}^{\ell}\right)$ (respectively $\left.\tilde{\Sigma}_{L}^{-\ell}=\mathbf{r}\left(\omega_{L} \times\left\{x_{3}=-\ell / 2\right\}\right)=\mathbf{r}\left(\Sigma_{L}^{-\ell}\right)\right)$. $\tilde{\Gamma}_{L}^{\ell}$ is the lateral boundary, $\tilde{\Gamma}_{L}^{\ell}=\mathbf{r}\left(\partial \omega_{L} \times\right.$ $(-\ell / 2, \ell / 2))=\mathbf{r}\left(\Gamma_{L}^{\ell}\right)$. The small parameter $\varepsilon$ in the problem is the ratio between the shell thickness and the characteristic horizontal length $\varepsilon=\ell / L \ll 1$.

Table 1: Parameter and unknowns description

\begin{tabular}{|l|l|}
\hline \hline$S Y M B O L$ & QUANTITY \\
\hline$\mu$ & shear modulus (Lamé's second parameter) \\
\hline$\lambda$ & Lamé's first parameter \\
\hline$\beta_{G}$ & inverse of Biot's modulus \\
\hline$\alpha$ & effective stress coefficient \\
\hline$k$ & permeability \\
\hline$\eta$ & viscosity \\
\hline$L$ and $\ell$ & midsurface length and shell width, respectively \\
\hline$\varepsilon=\ell / L$ & small parameter \\
\hline$T=\eta L_{c}^{2} /(k \mu)$ & characteristic Terzaghi's time \\
\hline$U$ & characteristic displacement \\
\hline$P=U \mu / L$ & characteristic fluid pressure \\
\hline $\mathbf{u}=\left(u_{1}, u_{2}, u_{3}\right)$ & solid phase displacement \\
\hline$p$ & pressure \\
\hline
\end{tabular}

We note that Biot's diphasic equations describe behavior of the system at so called Terzaghi's time scale $T=\eta L_{c}^{2} /(k \mu)$, where $L_{c}$ is the characteristic domain size, $\eta$ is dynamic viscosity, $k$ is permeability and $\mu$ is the shear modulus. For the list of all parameters see Table 1.

Similarly as in [20], we chose as the characteristic length $L_{c}=\ell$, which leads to the Taber-Terzaghi transversal time $T_{t a b}=\eta \ell^{2} /(k \mu)$. Another possibility was to choose the longitudinal time scaling with $T_{\text {long }}=\eta L^{2} /(k \mu)$. It would lead to different scaling in (2.8) and the dimensionless permeability coefficient in (3.3) would not be $\varepsilon^{2}$ but 1 . In the context of thermoelasticity, one has the same equations and Blanchard and Francfort rigorously derived in [2] the corresponding thermoelastic plate equations. We note that considering the longitudinal time scale yields the effective model where the pressure (i.e. the temperature in thermoelasticity) is decoupled from the flexion.

Then the quasi-static Biot equations for the poroelastic body $\tilde{\Omega}_{L}^{\ell}$ take the following dimensional form:

$$
\begin{gathered}
\tilde{\sigma}=2 \mu \mathbf{e}(\tilde{\mathbf{u}})+(\lambda \operatorname{div} \tilde{\mathbf{u}}-\alpha \tilde{p}) \mathbf{I} \text { in } \tilde{\Omega}_{L}^{\ell}, \\
-\operatorname{div} \tilde{\sigma}=-\mu \triangle \tilde{\mathbf{u}}-(\lambda+\mu) \nabla \operatorname{div} \tilde{\mathbf{u}}+\alpha \nabla \tilde{p}=0 \text { in } \tilde{\Omega}_{L}^{\ell}, \\
\frac{\partial}{\partial t}\left(\beta_{G} \tilde{p}+\alpha \operatorname{div} \tilde{\mathbf{u}}\right)-\frac{k}{\eta} \triangle \tilde{p}=0 \text { in } \tilde{\Omega}_{L}^{\ell} .
\end{gathered}
$$


Note that $\mathbf{e}(\mathbf{u})=\operatorname{sym} \nabla \mathbf{u}$ and $\tilde{\sigma}$ is the stress tensor. All other quantities are defined in Table 1.

We impose a given contact force $\tilde{\sigma} \boldsymbol{\nu}=\tilde{\mathcal{P}}_{L}^{ \pm \ell}$ and a given normal flux $-\frac{k}{\eta} \frac{\partial \tilde{p}}{\partial x_{3}}=\tilde{V}_{L}$ at $x_{3}= \pm \ell / 2$. At the lateral boundary $\tilde{\Gamma}^{\ell}$ we impose a zero displacement and a zero normal flux. Here $\boldsymbol{\nu}$ is the outer unit normal at the boundary. At initial time $t=0$ we prescribe the initial pressure $\tilde{p}_{L, i n}^{\ell}$.

Our goal is to extend the elliptic membrane shell justification by Ciarlet, Lods et al and by Dauge et al to the poroelastic case. Thus in the sequel we assume that the middle surface is elliptic (Gaussian curvature (product of principal curvatures) is positive at all points) and that the shell is clamped at its entire boundary.

We announce briefly the differential equations of the membrane poroelastic shell in dimensional form.

Effective dimensional equations:

The model is given in terms of $\mathbf{u}^{\mathrm{eff}}: \omega_{L} \rightarrow \mathbb{R}^{3}$ which is the vector of components of the displacement of the middle surface of the shell in the contravariant basis and $p^{\text {eff }}: \Omega_{L}^{\ell} \rightarrow \mathbb{R}$ which is the pressure in the $3 \mathrm{D}$ shell. Let us denote the stress tensor due to the variation in pore pressure across the shell thickness by

$$
\mathbf{n}=\ell \tilde{\mathcal{C}}^{c}\left(\mathbf{A}^{c} \boldsymbol{\gamma}\left(\mathbf{u}^{\mathrm{eff}}\right)\right) \mathbf{A}^{c}-\frac{2 \mu \alpha}{\lambda+2 \mu} \int_{-\ell / 2}^{\ell / 2} p^{\mathrm{eff}} d y_{3} \mathbf{A}^{c},
$$

where $\gamma(\cdot)$ is given by $(2.5)$ and $\tilde{\mathcal{C}}^{c}$ is the elasticity tensor, usually appearing in the classical shell theories, given by

$$
\tilde{\mathcal{C}}^{c} \mathbf{E}=2 \mu \frac{\lambda}{\lambda+2 \mu} \operatorname{tr}(\mathbf{E}) \mathbf{I}+2 \mu \mathbf{E}, \quad \mathbf{E} \in \mathbb{R}_{\text {sym }}^{2 \times 2} .
$$

Then the model in the differential formulation reads as follows:

$$
\begin{gathered}
-\left.\sum_{\beta=1}^{2} n_{\alpha \beta}\right|_{\beta}=\left(\mathcal{P}_{L}^{+\ell}\right)_{\alpha}+\left(\mathcal{P}_{L}^{-\ell}\right)_{\alpha} \text { in } \omega_{L}, \quad \alpha=1,2, \\
-\sum_{\alpha, \beta=1}^{2} b_{\alpha \beta} n_{\alpha \beta}=\left(\mathcal{P}_{L}^{+\ell}\right)_{3}+\left(\mathcal{P}_{L}^{-\ell}\right)_{3} \text { in } \omega_{L}, \\
u_{\alpha}^{\mathrm{eff}}=0, \alpha=1,2, \quad \text { on } \partial \omega_{L}, \quad \text { for every } t \in(0, T), \\
\left(\beta_{G}+\frac{\alpha^{2}}{\lambda+2 \mu}\right) \frac{\partial p^{\mathrm{eff}}}{\partial t}+\alpha \frac{2 \mu}{\lambda+2 \mu} \mathbf{A}^{c}: \gamma\left(\frac{\partial \mathbf{u}^{\mathrm{eff}}}{\partial t}\right)-\frac{k}{\eta} \frac{\partial^{2} p^{\mathrm{eff}}}{\partial\left(y_{3}\right)^{2}}=0 \\
\frac{k}{\eta} \frac{\partial p^{\mathrm{eff}}}{\partial y_{3}}=-V_{L}, \quad \text { on }(0, T) \times \omega_{L} \times(-\ell / 2, \ell / 2), \\
p^{\mathrm{eff}}=p_{L, \text { in }}^{\ell} \quad \text { given at } t=0 .
\end{gathered}
$$

Here $\left(\mathcal{P}_{L}^{ \pm \ell}\right)_{i}, i=1,2,3$ are components of the contact force $\tilde{\mathcal{P}}_{L}^{ \pm \ell} \circ \boldsymbol{r}$ at $\Sigma_{L}^{ \pm \ell}$ in the covariant basis, $V_{L}=\tilde{V}_{L} \circ \boldsymbol{X}, p_{L, \text { in }}^{\ell}=\tilde{p}_{L, \text { in }}^{\ell} \circ \boldsymbol{r}$. Thus, the poroelastic elliptic membrane shell model in the differential formulation is given for unknowns $\left\{\mathbf{n}, \mathbf{u}^{\text {eff }}, p^{\text {eff }}\right\}$ and by equations (2.9), (2.10) and (2.11). The components of $\mathbf{n}$ are the contact forces. The first two equations in (2.10) can be found in the differential equation of the elliptic membrane shell model (see [5, Theorem 4.5-1]). The first equation in (2.11) is the evolution equation for the effective pressure with associated boundary and initial conditions in the remaining part of (2.11). 
In the case of the classical theory of the purely elastic shell, we recall that, in addition to already quoted articles and books by Ciarlet and al, there is a huge literature, with both mathematical and engineering approaches (see e.g. [1], [12], [18], [25] and references therein).

In Section 3 we present the dimensionless form of the problem, then recall existence and uniqueness result of the smooth solution for the starting problem, rewrite the problem in curvilinear coordinates and rescale the problem on the domain $\Omega=\omega \times(-1 / 2,1 / 2)$. At the end of this section the main convergence results are formulated. In Section 4 we study the a priori estimates for the family of solutions. Then in Section 5 the convergence (including strong) of the solutions to the rescaled problem, is studied as $\varepsilon \rightarrow 0$. In Appendix we give the limit model written for a part of the spherical surface. Finally, the radially symmetric effective equations the problem on the whole sphere are derived and the result is compared with the one in [31].

\section{Problem setting in curvilinear coordinates and the main results}

\subsection{Dimensionless equations}

We introduce the dimensionless unknowns and variable by setting

$$
\begin{gathered}
\beta=\beta_{G} \mu ; \quad P=\frac{\mu U}{L} ; \quad U \tilde{\mathbf{u}}^{\varepsilon}=\tilde{\mathbf{u}} ; \quad T=\frac{\eta \ell^{2}}{k \mu} ; \quad \tilde{\lambda}=\frac{\lambda}{\mu} ; \\
P \tilde{p}^{\varepsilon}=\tilde{p} ; \quad \tilde{y} L=y ; \quad \tilde{x}_{3} L=x_{3} ; \quad \tilde{\mathbf{r}} L=\mathbf{r} ; \quad \tilde{\mathbf{X}} L=\mathbf{X} ; \quad \tilde{t} T=t ; \quad \tilde{\sigma}^{\varepsilon} \frac{\mu U}{L}=\tilde{\sigma} .
\end{gathered}
$$

After dropping wiggles in the coordinates and in the time, the system (2.6)-(2.8) becomes

$$
\begin{aligned}
& -\operatorname{div} \tilde{\sigma}^{\varepsilon}=-\triangle \tilde{\mathbf{u}}^{\varepsilon}-\tilde{\lambda} \nabla \operatorname{div} \tilde{\mathbf{u}}^{\varepsilon}+\alpha \nabla \tilde{p}^{\varepsilon}=0 \text { in }(0, T) \times \tilde{\Omega}^{\varepsilon}, \\
& \tilde{\sigma}^{\varepsilon}=2 \mathbf{e}\left(\tilde{\mathbf{u}}^{\varepsilon}\right)+\left(\tilde{\lambda} \operatorname{div} \tilde{\mathbf{u}}^{\varepsilon}-\alpha \tilde{p}^{\varepsilon}\right) \mathbf{I} \text { in }(0, T) \times \tilde{\Omega}^{\varepsilon}, \\
& \frac{\partial}{\partial t}\left(\beta \tilde{p}^{\varepsilon}+\alpha \operatorname{div} \tilde{\mathbf{u}}^{\varepsilon}\right)-\varepsilon^{2} \triangle \tilde{p}^{\varepsilon}=0 \quad \text { in }(0, T) \times \tilde{\Omega}^{\varepsilon},
\end{aligned}
$$

where $\tilde{\mathbf{u}}^{\varepsilon}=\left(\tilde{u}_{1}^{\varepsilon}, \tilde{u}_{2}^{\varepsilon}, \tilde{u}_{3}^{\varepsilon}\right)$ denotes the dimensionless displacement field and $\tilde{p}^{\varepsilon}$ the dimensionless pressure. We study a shell $\tilde{\Omega}^{\varepsilon}$ with thickness $\varepsilon=\ell / L$ and section $\omega=\omega_{L} / L$. It is described by

$$
\tilde{\Omega}^{\varepsilon}=\frac{1}{L} \mathbf{r}\left(\left\{\left(x_{1}, x_{2}, x_{3}\right) / L \in \omega \times(-\varepsilon / 2, \varepsilon / 2)\right\}\right)=\mathbf{r}\left(\Omega_{L}^{\ell}\right)=\tilde{\Omega}_{L}^{\ell} / L,
$$

$\tilde{\Sigma}_{+}^{\varepsilon}\left(\right.$ respectively $\left.\tilde{\Sigma}_{-}^{\varepsilon}\right)$ is the upper face (respectively the lower face) of the shell $\tilde{\Omega}^{\varepsilon} . \tilde{\Gamma}^{\varepsilon}$ is the lateral boundary, $\tilde{\Gamma}^{\varepsilon}=\tilde{\Gamma}_{L}^{\ell} / L$.

We suppose that a given dimensionless traction force is applied on $\tilde{\Sigma}_{+}^{\varepsilon} \cup \tilde{\Sigma}_{-}^{\varepsilon}$ and impose the shell is clamped on $\tilde{\Gamma}^{\varepsilon}$ :

$$
\begin{gathered}
\tilde{\sigma}^{\varepsilon} \boldsymbol{\nu}=\left(2 \mathbf{e}\left(\tilde{\mathbf{u}}^{\varepsilon}\right)-\alpha \tilde{p}^{\varepsilon} I+\tilde{\lambda}\left(\operatorname{div} \tilde{\mathbf{u}}^{\varepsilon}\right) I\right) \boldsymbol{\nu}=\varepsilon \tilde{\mathcal{P}}_{ \pm} \text {on } \tilde{\Sigma}_{ \pm}^{\varepsilon}, \\
\tilde{\mathbf{u}}^{\varepsilon}=0, \quad \text { on } \tilde{\Gamma}^{\varepsilon} .
\end{gathered}
$$

For the pressure $\tilde{p}^{\varepsilon}$, at the lateral boundary $\tilde{\Gamma}^{\varepsilon}$ the zero inflow/outflow flux is imposed:

$$
-\nabla \tilde{p}^{\varepsilon} \cdot \boldsymbol{\nu}=0 .
$$

and at $\tilde{\Sigma}_{ \pm}^{\varepsilon}$, we set

$$
-\varepsilon^{2} \nabla \tilde{p}^{\varepsilon} \cdot \boldsymbol{\nu}= \pm \varepsilon \tilde{V} .
$$

Finally, we need an initial condition for $\tilde{p}^{\varepsilon}$ at $t=0$,

$$
\tilde{p}^{\varepsilon}\left(x_{1}, x_{2}, x_{3}, 0\right)=\tilde{p}_{\text {in }}\left(x_{1}, x_{2}\right) \quad \text { in } \tilde{\Omega}^{\varepsilon} .
$$

The difference here, with respect to flexural shell case ([22]), is that the contact loads in (3.4) and filtration velocity in (3.7) are differently scaled. 
Remark 1. We recall that in the flexural shell case contact loads were assumed to behave like $\varepsilon^{3} \tilde{\mathcal{P}}_{ \pm}$ and the normal boundary filtration velocity by $-\varepsilon^{2} \nabla \tilde{p}^{\varepsilon} \cdot \boldsymbol{\nu}= \pm \varepsilon^{2} \tilde{V}$.

Let $\mathcal{V}\left(\tilde{\Omega}^{\varepsilon}\right)=\left\{\tilde{\mathbf{v}} \in H^{1}\left(\tilde{\Omega}^{\varepsilon} ; \mathbb{R}^{3}\right):\left.\tilde{\mathbf{v}}\right|_{\tilde{\Gamma}^{\varepsilon}}=0\right\}$. Then the weak formulation corresponding to (3.1)(3.8) is given by

Find $\tilde{\mathbf{u}}^{\varepsilon} \in H^{1}\left(0, T, \mathcal{V}\left(\tilde{\Omega}^{\varepsilon}\right)\right), \tilde{p}^{\varepsilon} \in H^{1}\left(0, T ; H^{1}\left(\tilde{\Omega}^{\varepsilon}\right)\right)$ such that it holds

$$
\begin{aligned}
& \int_{\tilde{\Omega}^{\varepsilon}} 2 \mathbf{e}\left(\tilde{\mathbf{u}}^{\varepsilon}\right): \mathbf{e}(\tilde{\mathbf{v}}) d x+\tilde{\lambda} \int_{\tilde{\Omega}^{\varepsilon}} \operatorname{div} \tilde{\mathbf{u}}^{\varepsilon} \operatorname{div} \tilde{\mathbf{v}} d x-\alpha \int_{\tilde{\Omega}^{\varepsilon}} \tilde{p}^{\varepsilon} \operatorname{div} \tilde{\mathbf{v}} d x \\
& =\int_{\tilde{\Sigma}_{+}^{\varepsilon}} \varepsilon \tilde{\mathcal{P}}_{+} \cdot \tilde{\mathbf{v}} d s+\int_{\tilde{\Sigma}_{-}^{\varepsilon}} \varepsilon \tilde{\mathcal{P}}_{-} \cdot \tilde{\mathbf{v}} d s, \quad \text { for every } \tilde{\mathbf{v}} \in \mathcal{V}\left(\tilde{\Omega}^{\varepsilon}\right) \text { and } t \in(0, T), \\
& \beta \int_{\tilde{\Omega}^{\varepsilon}} \partial_{t} \tilde{p}^{\varepsilon} \tilde{q} d x+\int_{\tilde{\Omega}^{\varepsilon}} \alpha \operatorname{div} \partial_{t} \tilde{\mathbf{u}}^{\varepsilon} \tilde{q} d x+\varepsilon^{2} \int_{\tilde{\Omega}^{\varepsilon}} \nabla \tilde{p}^{\varepsilon} \cdot \nabla \tilde{q} d x \\
& =\varepsilon \int_{\tilde{\Sigma}_{-}^{\varepsilon}} \tilde{V} \tilde{q} d s-\varepsilon \int_{\tilde{\Sigma}_{+}^{\varepsilon}} \tilde{V} \tilde{q} d s, \quad \text { for every } \tilde{q} \in H^{1}\left(\tilde{\Omega}^{\varepsilon}\right) \text { and } t \in(0, T), \\
& \left.\tilde{p}^{\varepsilon}\right|_{\{t=0\}}=\tilde{p}_{\text {in }}, \quad \text { in } \tilde{\Omega}^{\varepsilon} .
\end{aligned}
$$

Note that for two $3 \times 3$ matrices $A$ and $B$ the Frobenius scalar product is denoted by $A: B=$ $\operatorname{tr}\left(A B^{T}\right)$.

\subsection{Existence and uniqueness for the $\varepsilon$-problem}

In this subsection the existence and uniqueness of a solution $\left\{\tilde{\mathbf{u}}^{\varepsilon}, \tilde{p}^{\varepsilon}\right\} \in H^{1}\left(0, T ; \mathcal{V}\left(\tilde{\Omega}^{\varepsilon}\right)\right) \times H^{1}\left(0, T ; H^{1}\left(\tilde{\Omega}^{\varepsilon}\right)\right)$ to problem (3.9)-(3.11) is recalled. We follow [20] and get

Proposition 2. Let us suppose

$$
\tilde{p}_{\text {in }} \in H_{0}^{2}\left(\tilde{\Omega}^{\varepsilon}\right), \mathcal{P}_{ \pm} \in H^{2}\left(0, T ; L^{2}\left(\omega ; \mathbb{R}^{3}\right)\right) \text { and } \tilde{V} \in H^{1}\left(0, T ; L^{2}(\omega)\right),\left.\tilde{V}\right|_{\{t=0\}}=0 .
$$

Then problem (3.9)-(3.11) has a unique solution $\left.\left\{\tilde{\mathbf{u}}^{\varepsilon}, \tilde{p}^{\varepsilon}\right\} \in H^{1}\left(0, T ; \mathcal{V}\left(\tilde{\Omega}^{\varepsilon}\right)\right)\right) \times H^{1}\left(0, T ; H^{1}\left(\tilde{\Omega}^{\varepsilon}\right)\right)$.

\subsection{Problem in Curvilinear Coordinates and the Scaled Problem}

In this section we introduce the formulation of the problem in curvilinear coordinates. The formulation is the same as in Subsection 3.3 in [22, pages 371-374] without the rescaled problem in (3.18). For completeness and for the comfort of the reader we repeat it here.

Our goal is to find the limits of the solutions of problem (3.9)-(3.11) when $\varepsilon$ tends to zero. It is known from similar considerations made for classical shells that asymptotic behavior of the longitudinal and transverse displacements of the elastic body is different. The same effect is expected in the present setting. Therefore we need to consider asymptotic behavior of the local components of the displacement $\tilde{\mathbf{u}}^{\varepsilon}$. It can be done in many ways, but in order to preserve some important properties of bilinear forms, such as positive definiteness and symmetry, we rewrite the three-dimensional equations in curvilinear coordinates defined by $\mathbf{r}$. Then we formulate equivalent problems posed on the domain independent of $\varepsilon$. We essentially follow the analogous section from [22]. Nevertheless, it should be noted that the pressure scaling is different.

Let $\tilde{\mathbf{r}}(x / L)=\mathbf{r}(x) / L, x \in \Omega_{L}^{\ell}$. The covariant basis of the shell $\tilde{\Omega^{\varepsilon}}$, which is the three-dimensional manifold parameterized by $\mathbf{r}$, is defined by

$$
\mathbf{g}_{i}^{\varepsilon}=\partial_{i} \mathbf{r}: \Omega^{\varepsilon} \rightarrow \mathbb{R}^{3}, \quad i=1,2,3 .
$$


Vectors $\left\{\mathbf{g}_{1}^{\varepsilon}, \mathbf{g}_{2}^{\varepsilon}, \mathbf{g}_{3}^{\varepsilon}\right\}$ are given by

$$
\begin{aligned}
& \mathbf{g}_{1}^{\varepsilon}=\mathbf{a}_{1}(y)+x_{3} \partial_{y_{1}} \mathbf{a}_{3}(y), \\
& \mathbf{g}_{2}^{\varepsilon}=\mathbf{a}_{2}(y)+x_{3} \partial_{y_{2}} \mathbf{a}_{3}(y), \\
& \mathbf{g}_{3}^{\varepsilon}=\mathbf{a}_{3}(y) .
\end{aligned}
$$

Vectors $\left\{\mathbf{g}^{1, \varepsilon}, \mathbf{g}^{2, \varepsilon}, \mathbf{g}^{3, \varepsilon}\right\}$ satisfying

$$
\mathbf{g}^{j, \varepsilon} \cdot \mathbf{g}_{i}^{\varepsilon}=\delta_{i j} \text { on } \bar{\Omega}^{\varepsilon}, \quad i, j=1,2,3,
$$

where $\delta_{i j}$ is the Kronecker symbol, form the contravariant basis on $\overline{\tilde{\Omega}}$. The contravariant metric tensor $\mathbf{G}^{c, \varepsilon}=\left(g^{i j, \varepsilon}\right)$, the covariant metric tensor $\mathbf{G}_{c}^{\varepsilon}=\left(g_{i j}^{\varepsilon}\right)$ and the Christoffel symbols $\Gamma_{j k}^{i, \varepsilon}$ of the shell $\bar{\Omega}^{\varepsilon}$ are defined by

$$
g^{i j, \varepsilon}=\mathbf{g}^{i, \varepsilon} \cdot \mathbf{g}^{j, \varepsilon}, \quad g_{i j}^{\varepsilon}=\mathbf{g}_{i}^{\varepsilon} \cdot \mathbf{g}_{j}^{\varepsilon}, \quad \Gamma_{j k}^{i, \varepsilon}=\mathbf{g}^{i, \varepsilon} \cdot \partial_{j} \mathbf{g}_{k}^{\varepsilon} \text { on } \bar{\Omega}^{\varepsilon}, \quad i, j, k=1,2,3 .
$$

We set

$$
\boldsymbol{\Gamma}^{i, \varepsilon}=\left(\Gamma_{j k}^{i, \varepsilon}\right)_{j, k=1, \ldots, 3} \quad \text { and } \quad \tilde{\gamma}_{\varepsilon}(\mathbf{v})=\frac{1}{2}\left(\nabla \mathbf{v}+\nabla \mathbf{v}^{T}\right)-\sum_{i=1}^{3} v_{i} \boldsymbol{\Gamma}^{i, \varepsilon}
$$

Let $g^{\varepsilon}=\operatorname{det} \mathbf{G}_{c}^{\varepsilon}$. Until now we were using the canonical basis $\left\{\mathbf{e}_{1}, \mathbf{e}_{2}, \mathbf{e}_{3}\right\}$, for $\mathbb{R}^{3}$. Now the displacement is rewritten in the contravariant basis,

$$
\tilde{\mathbf{u}}^{\varepsilon} \circ \mathbf{r}\left(y_{1}, y_{2}, x_{3}\right)=\sum_{i=1}^{3} \tilde{u}_{i}^{\varepsilon} \circ \mathbf{r}\left(y_{1}, y_{2}, x_{3}\right) \mathbf{e}_{i}=\sum_{i=1}^{3} u_{i}^{\varepsilon}\left(y_{1}, y_{2}, x_{3}\right) \mathbf{g}^{i, \varepsilon}\left(y_{1}, y_{2}, x_{3}\right), \quad \tilde{\mathbf{v}} \circ \mathbf{r}=\sum_{i=1}^{3} v_{i} \mathbf{g}^{i, \varepsilon},
$$

while for scalar fields we just change the coordinates

$$
\tilde{p}^{\varepsilon} \circ \mathbf{r}=p^{\varepsilon}, \quad \tilde{q} \circ \mathbf{r}=q, \quad \tilde{V} \circ \mathbf{r}=V, \quad \tilde{p}_{\text {in }} \circ \mathbf{r}=p_{\text {in } n},
$$

on $\bar{\Omega}^{\varepsilon}$. The contact forces are rewritten in the covariant basis of the shell

$$
\tilde{\mathcal{P}}_{ \pm} \circ \mathbf{r}=\sum_{i=1}^{3}\left(\mathcal{P}_{ \pm}\right)_{i} \mathbf{g}_{i}^{\varepsilon} \text { on } \Sigma_{ \pm}^{\varepsilon} .
$$

New vector functions are defined by

$$
\mathbf{u}^{\varepsilon}=u_{i}^{\varepsilon} \mathbf{e}_{i}, \quad \mathbf{v}=v_{i} \mathbf{e}_{i}, \quad \mathcal{P}_{ \pm}=\left(\mathcal{P}_{ \pm}\right)_{i} \mathbf{e}_{i} .
$$

Note that $u^{\varepsilon}{ }_{i}$ are not components of the physical displacement. They are just intermediate functions which will be used to reconstruct $\tilde{\mathbf{u}}^{\varepsilon}$. The corresponding function space to $\mathcal{V}\left(\tilde{\Omega}^{\varepsilon}\right)$ is the space

$$
\mathcal{V}\left(\Omega^{\varepsilon}\right)=\left\{\mathbf{v} \in H^{1}\left(\Omega^{\varepsilon}\right)^{3}:\left.\mathbf{v}\right|_{\Gamma^{\varepsilon}}=0\right\} .
$$

Let $\mathbf{Q}^{\varepsilon}=(\nabla \mathbf{r})^{-T}=\left(\mathbf{g}_{1}^{\varepsilon} \mathbf{g}_{2}^{\varepsilon} \mathbf{g}_{3}^{\varepsilon}\right)^{-T}=\left(\mathbf{g}^{1, \varepsilon} \mathbf{g}^{2, \varepsilon} \mathbf{g}^{3, \varepsilon}\right)$ and let

$$
\mathcal{C} \mathbf{E}=\tilde{\lambda}(\operatorname{tr} \mathbf{E}) \mathbf{I}+2 \mathbf{E}, \quad \text { for all } \quad \mathbf{E} \in \mathbb{R}_{\text {sym }}^{3 \times 3} .
$$

Then the problem (3.9)-(3.11) can be written as

$$
\begin{aligned}
& \int_{\Omega^{\varepsilon}} \mathcal{C}\left(\mathbf{Q}^{\varepsilon} \tilde{\boldsymbol{\gamma}}_{\varepsilon}\left(\mathbf{u}^{\varepsilon}\right)\left(\mathbf{Q}^{\varepsilon}\right)^{T}\right):\left(\mathbf{Q}^{\varepsilon} \tilde{\boldsymbol{\gamma}}_{\varepsilon}(\mathbf{v})\left(\mathbf{Q}^{\varepsilon}\right)^{T}\right) \sqrt{g^{\varepsilon}} d y-\alpha \int_{\Omega^{\varepsilon}} p^{\varepsilon} \operatorname{tr}\left(\mathbf{Q}^{\varepsilon} \tilde{\gamma}_{\varepsilon}(\mathbf{v})\left(\mathbf{Q}^{\varepsilon}\right)^{T}\right) \sqrt{g^{\varepsilon}} d y \\
& \quad=\varepsilon \int_{\Sigma_{+}^{\varepsilon}} \mathcal{P}_{+} \cdot \mathbf{v} \sqrt{g^{\varepsilon}} d s+\varepsilon \int_{\Sigma_{-}^{\varepsilon}} \mathcal{P}_{-} \cdot \mathbf{v} \sqrt{g^{\varepsilon}} d s, \quad \mathbf{v} \in \mathcal{V}\left(\Omega^{\varepsilon}\right), \text { a.e. } t \in[0, T], \\
& \int_{\Omega^{\varepsilon}} \beta \frac{\partial p^{\varepsilon}}{\partial t} q \sqrt{g^{\varepsilon}} d y+\int_{\Omega^{\varepsilon}} \alpha \frac{\partial}{\partial t} \operatorname{tr}\left(\mathbf{Q}^{\varepsilon} \tilde{\gamma}_{\varepsilon}\left(\mathbf{u}^{\varepsilon}\right)\left(\mathbf{Q}^{\varepsilon}\right)^{T}\right) q \sqrt{g^{\varepsilon}} d y \\
& \quad+\varepsilon^{2} \int_{\Omega^{\varepsilon}} \mathbf{Q}^{\varepsilon} \nabla p^{\varepsilon} \cdot \mathbf{Q}^{\varepsilon} \nabla q \sqrt{g^{\varepsilon}} d y=\varepsilon \int_{\Sigma_{-}^{\varepsilon}} V q \sqrt{g^{\varepsilon}} d s-\varepsilon \int_{\Sigma_{+}^{\varepsilon}} V q \sqrt{g^{\varepsilon}} d s, \\
& \quad q \in H^{1}\left(\Omega^{\varepsilon}\right), \text { a.e. } t \in[0, T], \\
& p^{\varepsilon}=p_{\text {in }}, \quad \text { for } t=0 .
\end{aligned}
$$


This is the problem in curvilinear coordinates.

Problems for all $\tilde{\mathbf{u}}^{\varepsilon}, \tilde{p}^{\varepsilon}$ and $\mathbf{u}^{\varepsilon}, p^{\varepsilon}$ are posed on $\varepsilon$-dependent domains. In the sequel we follow the idea from Ciarlet, Destuynder [6] and rewrite (3.15) on the canonical domain independent of $\varepsilon$. As a consequence, the coefficients of the resulting weak formulation will depend on $\varepsilon$ explicitly.

Let $\Omega=\omega \times(-1 / 2,1 / 2)$ and let $\mathbf{R}^{\varepsilon}: \bar{\Omega} \rightarrow \bar{\Omega}^{\varepsilon}$ be defined by

$$
\mathbf{R}^{\varepsilon}(z)=\left(z^{1}, z^{2}, \varepsilon z^{3}\right), \quad z \in \Omega, \varepsilon \in\left(0, \varepsilon_{0}\right) .
$$

By $\Sigma_{ \pm}=\omega \times\{ \pm 1 / 2\}$ we denote the upper and lower face of $\Omega$. Let $\Gamma=\partial \omega \times(-1 / 2,1 / 2)$. To the functions $\mathbf{u}^{\varepsilon}, p^{\varepsilon}, g^{\varepsilon}, \mathbf{g}_{i}^{\varepsilon}, \mathbf{g}^{\varepsilon, i}, \mathbf{Q}^{\varepsilon}, \Gamma_{j k}^{i, \varepsilon}, i, j, k=1,2,3$ defined on $\bar{\Omega}^{\varepsilon}$ we associate the functions $\mathbf{u}(\varepsilon)$, $p(\varepsilon), g(\varepsilon), \mathbf{g}_{i}(\varepsilon), \mathbf{g}^{i}(\varepsilon), \mathbf{Q}(\varepsilon), \Gamma_{i j}^{i}(\varepsilon), i, j, k=1,2,3$ defined on $\bar{\Omega}$ by composition with $\mathbf{R}^{\varepsilon}$. Let us also define

$$
\mathcal{V}(\Omega)=\left\{\mathbf{v}=\left(v_{1}, v_{2}, v_{3}\right) \in H^{1}\left(\Omega ; \mathbb{R}^{3}\right):\left.\mathbf{v}\right|_{\Gamma}=0\right\} .
$$

Then the problem (3.15) can be written as

$$
\begin{aligned}
& \varepsilon \int_{\Omega} \mathcal{C}\left(\mathbf{Q}(\varepsilon) \boldsymbol{\gamma}^{\varepsilon}(\mathbf{u}(\varepsilon)) \mathbf{Q}(\varepsilon)^{T}\right):\left(\mathbf{Q}(\varepsilon) \boldsymbol{\gamma}^{\varepsilon}(\mathbf{v}) \mathbf{Q}(\varepsilon)^{T}\right) \sqrt{g(\varepsilon)} d z \\
& \quad-\varepsilon \alpha \int_{\Omega} p(\varepsilon) \operatorname{tr}\left(\mathbf{Q}(\varepsilon) \boldsymbol{\gamma}^{\varepsilon}(\mathbf{v}) \mathbf{Q}(\varepsilon)^{T}\right) \sqrt{g(\varepsilon)} d z \\
& =\varepsilon \int_{\Sigma_{ \pm}} \mathcal{P}_{ \pm} \cdot \mathbf{v} \sqrt{g(\varepsilon)} d s, \quad \mathbf{v} \in \mathcal{V}(\Omega) \text {, a.e. } t \in[0, T], \\
& \varepsilon \int_{\Omega} \beta \frac{\partial p(\varepsilon)}{\partial t} q \sqrt{g(\varepsilon)} d z+\varepsilon \int_{\Omega} \alpha \frac{\partial}{\partial t} \operatorname{tr}\left(\mathbf{Q}(\varepsilon) \boldsymbol{\gamma}^{\varepsilon}(\mathbf{u}(\varepsilon)) \mathbf{Q}(\varepsilon)^{T}\right) q \sqrt{g(\varepsilon)} d z \\
& \quad+\varepsilon^{3} \int_{\Omega} \mathbf{Q}(\varepsilon) \nabla^{\varepsilon} p(\varepsilon) \cdot \mathbf{Q}(\varepsilon) \nabla^{\varepsilon} q \sqrt{g(\varepsilon)} d z \\
& =\mp \varepsilon \int_{\Sigma_{ \pm}} V q \sqrt{g(\varepsilon)} d s, \quad q \in H^{1}(\Omega), \text { a.e. } t \in[0, T], \\
& p(\varepsilon)=p_{\text {in }}, \quad \text { for } t=0 .
\end{aligned}
$$

Here

$$
\begin{aligned}
& \boldsymbol{\gamma}^{\varepsilon}(\mathbf{v})=\frac{1}{\varepsilon} \boldsymbol{\gamma}_{z}(\mathbf{v})+\boldsymbol{\gamma}_{y}(\mathbf{v})-\sum_{i=1}^{3} v_{i} \boldsymbol{\Gamma}^{i}(\varepsilon) \\
& \gamma_{z}(\mathbf{v})=\left[\begin{array}{ccc}
0 & 0 & \frac{1}{2} \partial_{3} v_{1} \\
0 & 0 & \frac{1}{2} \partial_{3} v_{2} \\
\frac{1}{2} \partial_{3} v_{1} & \frac{1}{2} \partial_{3} v_{2} & \partial_{3} v_{3}
\end{array}\right], \gamma_{y}(\mathbf{v})=\left[\begin{array}{ccc}
\partial_{1} v_{1} & \frac{1}{2}\left(\partial_{2} v_{1}+\partial_{1} v_{2}\right) & \frac{1}{2} \partial_{1} v_{3} \\
\frac{1}{2}\left(\partial_{2} v_{1}+\partial_{1} v_{2}\right) & \partial_{2} v_{2} & \frac{1}{2} \partial_{2} v_{3} \\
\frac{1}{2} \partial_{1} v_{3} & \frac{1}{2} \partial_{2} v_{3} & 0
\end{array}\right] \text {, } \\
& \nabla^{\varepsilon} q=\frac{1}{\varepsilon} \nabla_{z} q+\nabla_{y} q, \quad \nabla_{z} q=\left[\begin{array}{ccc}
0 & 0 & \partial_{3} q
\end{array}\right], \quad \nabla_{y} q=\left[\begin{array}{lll}
\partial_{1} q & \partial_{2} q & 0
\end{array}\right]
\end{aligned}
$$

and we have also used the notation

$$
\begin{aligned}
& \mp \int_{\Sigma_{ \pm}} V q \sqrt{g(\varepsilon)} d s=\int_{\Sigma_{-}} V q \sqrt{g(\varepsilon)} d s-\int_{\Sigma_{+}} V q \sqrt{g(\varepsilon)} d s \\
& \int_{\Sigma_{ \pm}} \mathcal{P}_{ \pm} \cdot \mathbf{v} \sqrt{g(\varepsilon)} d s=\int_{\Sigma_{+}} \mathcal{P}_{+} \cdot \mathbf{v} \sqrt{g(\varepsilon)} d s+\int_{\Sigma_{-}} \mathcal{P}_{-} \cdot \mathbf{v} \sqrt{g(\varepsilon)} d s .
\end{aligned}
$$

Remark 3. Existence and uniqueness of a smooth solution to problem (3.16) follows from Proposition 2 and the smoothness of the curvilinear coordinates transformation.

Also notice that in the present (elliptic membrane) case there is no rescaling of the pressure. It will appear to be of order one which is in contrast to the flexural shell case where it was of order $\varepsilon$. 


\subsection{Convergence results}

In the remainder of the paper we make the following assumptions

Assumption 4. For simplicity, we assume that $p_{\text {in }}=0$, that $V \in H^{1}\left(0, T ; L^{2}(\omega)\right),\left.V\right|_{\{t=0\}}=0$ and that $\mathcal{P}_{ \pm} \in H^{2}\left(0, T ; L^{2}\left(\omega ; \mathbb{R}^{3}\right)\right)$, with $\left.\mathcal{P}_{ \pm}\right|_{\{t=0\}}=0$.

To describe the limit problem we introduce the function space $\mathcal{V}_{M}(\omega)=H_{0}^{1}(\omega) \times H_{0}^{1}(\omega) \times L^{2}(\omega)$. Contrary to $\mathcal{V}_{F}(\omega)$, which is the function space for the flexural shell model, it is always non-trivial. The boundary value problem in $\Omega=\omega \times(-1 / 2,1 / 2)$ for the effective displacement and the effective pressure is given by:

find $\left\{\mathbf{u}, p^{0}\right\} \in C\left([0, T] ; \mathcal{V}_{M}(\omega) \times L^{2}(\Omega)\right), \partial_{z_{3}} p^{0} \in L^{2}((0, T) \times \Omega)$ satisfying the system

$$
\begin{gathered}
\int_{\omega} \tilde{\mathcal{C}}\left(\mathbf{A}^{c} \gamma(\mathbf{u})\right): \gamma(\mathbf{v}) \mathbf{A}^{c} \sqrt{a} d z_{1} d z_{2}-\frac{2 \alpha}{\tilde{\lambda}+2} \int_{\omega} \int_{-1 / 2}^{1 / 2} p^{0} d z_{3} \mathbf{A}^{c}: \gamma(\mathbf{v}) \sqrt{a} d z_{1} d z_{2} \\
=\int_{\omega}\left(\mathcal{P}_{+}+\mathcal{P}_{-}\right) \cdot \mathbf{v} \sqrt{a} d z_{1} d z_{2} ., \quad \mathbf{v} \in \mathcal{V}_{M}(\omega), \\
\int_{\Omega}\left(\beta+\frac{\alpha^{2}}{\tilde{\lambda}+2}\right) \frac{\partial p^{0}}{\partial t} q \sqrt{a} d z+\int_{\Omega} \alpha \frac{\partial}{\partial t}\left(\frac{2}{\tilde{\lambda}+2} \mathbf{A}^{c}: \gamma(\mathbf{u})\right) q \sqrt{a} d z+\int_{\Omega} \frac{\partial p^{0}}{\partial z_{3}} \frac{\partial q}{\partial z_{3}} \sqrt{a} d z \\
=\mp \int_{\Sigma_{ \pm}} V q \sqrt{a} d s, \quad q \in H^{1}(\Omega) . \\
p^{0}=0 \quad \text { at } t=0,
\end{gathered}
$$

where $\gamma(\cdot)$ is given by $(2.5)$ and

$$
\tilde{\mathcal{C}} \mathbf{E}=2 \frac{\tilde{\lambda}}{\tilde{\lambda}+2} \operatorname{tr}(\mathbf{E}) \mathbf{I}+2 \mathbf{E}, \quad \mathbf{E} \in \mathbb{R}_{\mathrm{sym}}^{2 \times 2} .
$$

Remark 5. We observe that, contrary to the effective flexural shell system from [22], problem (3.18)-(3.20) is of the second order.

Fundamental for the analysis of this model is the inequality of Korn's type on an elliptic surface, see [5, Theorem 2.7-3] or [7, Theorem 4.2].

Lemma 6. Let $\omega$ be a domain in $R^{2}$ and let $\boldsymbol{X} \in C^{2,1}\left(\bar{\omega} ; \mathbb{R}^{3}\right)$ be an injective mapping such that the two vectors $\mathbf{a}_{\alpha}=\partial_{\alpha} \boldsymbol{X}$ are linearly independent at all points of $\bar{\omega}$ and such that the surface $\boldsymbol{X}(\bar{\omega})$ is elliptic. Then there is $C_{M}>0$ such that

$$
\left\|v_{1}\right\|_{H^{1}(\omega)}^{2}+\left\|v_{2}\right\|_{H^{1}(\omega)}^{2}+\left\|v_{3}\right\|_{L^{2}(\omega)}^{2} \leq C_{M}\|\gamma(\mathbf{v})\|_{L^{2}\left(\omega ; \mathbb{R}^{3 \times 3}\right)}^{2}, \quad \mathbf{v} \in \mathcal{V}_{M}(\omega) .
$$

Proposition 7. Under Assumption 4, problem (3.18)-(3.20) has a unique solution $\left\{\mathbf{u}, p^{0}\right\}$ in the space $C\left([0, T] ; \mathcal{V}_{M}(\omega) \times L^{2}(\Omega)\right), \partial_{z_{3}} p^{0} \in L^{2}((0, T) \times \Omega)$ Furthermore, $\partial_{t} p^{0} \in L^{2}((0, T) \times \Omega)$ and $\partial_{t} \mathbf{u} \in L^{2}\left(0, T ; \mathcal{V}_{M}(\omega)\right)$.

Proof. We follow the proof of Proposition 4 from [22] and first prove that $\left\{\mathbf{u}, p^{0}\right\} \in C\left([0, T] ; \mathcal{V}_{M}(\omega) \times\right.$ $\left.L^{2}(\Omega)\right)$ and $\partial_{z_{3}} p^{0} \in L^{2}((0, T) \times \Omega)$ imply a higher regularity in time. Ideas are analogous but details of the calculations are different.

Next we take $q=\bar{q}\left(z_{1}, z_{2}\right), \bar{q} \in C^{\infty}(\bar{\omega})$ as a test function in (3.19). The time continuity and (3.19) yield

$$
\left(\beta+\frac{\alpha^{2}}{\tilde{\lambda}+2}\right) \int_{-1 / 2}^{1 / 2} p^{0} d z_{3}+\frac{2 \alpha}{\tilde{\lambda}+2} \mathbf{A}^{c}: \gamma(\mathbf{u})=0
$$


After inserting (3.22) into (3.18), it takes the form

$$
\begin{aligned}
& \int_{\omega} \tilde{\mathcal{C}}\left(\mathbf{A}^{c} \boldsymbol{\gamma}(\mathbf{u})\right): \boldsymbol{\gamma}(\mathbf{v}) \mathbf{A}^{c} \sqrt{a} d z_{1} d z_{2}+\frac{4 \alpha^{2}}{(\tilde{\lambda}+2)\left(\beta(\tilde{\lambda}+2)+\alpha^{2}\right)} \int_{\omega} \mathbf{A}^{c}: \boldsymbol{\gamma}(\mathbf{u}) \mathbf{A}^{c}: \boldsymbol{\gamma}(\mathbf{v}) \sqrt{a} d z_{1} d z_{2} \\
& =\int_{\omega}\left(\mathcal{P}_{+}+\mathcal{P}_{-}\right) \cdot \mathbf{v} \sqrt{a} d z_{1} d z_{2}, \quad \mathbf{v} \in \mathcal{V}_{M}(\omega) .
\end{aligned}
$$

The $H^{2}$-regularity in time of $\mathcal{P}_{ \pm}$allows taking time derivatives of equation (3.23) up to order 2 . It yields $\partial_{t} \mathbf{u} \in L^{2}\left(0, T ; \mathcal{V}_{M}(\omega)\right)$ and $\partial_{t t} \mathbf{u} \in L^{2}\left(0, T ; \mathcal{V}_{M}(\omega)\right)$. Hence $\partial_{t} \mathbf{u} \in H^{1}\left(0, T ; \mathcal{V}_{M}(\omega)\right)$. Note that contrary to the flexural case from the proof of Proposition 4 from [22], the equation for $\mathbf{u}$ is now of the 2nd order. For such $\mathbf{u}$ classical regularity theory for the second order linear parabolic equations applied at (3.19) implies $\partial_{t} p^{0} \in L^{2}((0, T) \times \Omega)$.

The existence and the uniqueness are based on the energy estimate. If we choose $\mathbf{v}=\frac{\partial \mathbf{u}}{\partial t}$ as a test function in (3.18) and $p^{0}$ as a test function in (3.19) and sum up the equations we obtain the equality

$$
\begin{aligned}
\frac{1}{2} \frac{d}{d t} & \left\{\int_{\omega} \tilde{\mathcal{C}}\left(\mathbf{A}^{c} \boldsymbol{\gamma}(\mathbf{u})\right): \boldsymbol{\gamma}(\mathbf{u}) \mathbf{A}^{c} \sqrt{a} d z_{1} d z_{2}+\int_{\Omega}\left(\beta+\frac{\alpha^{2}}{\tilde{\lambda}+2}\right)\left(p^{0}\right)^{2} \sqrt{a} d z\right. \\
& \left.-2 \int_{\omega}\left(\mathcal{P}_{+}+\mathcal{P}_{-}\right) \cdot \mathbf{u} \sqrt{a} d z_{1} d z_{2}\right\}+\int_{\Omega}\left(\frac{\partial p^{0}}{\partial z_{3}}\right)^{2} \sqrt{a} d z d t \\
= & -\int_{\omega} \partial_{t}\left(\mathcal{P}_{+}+\mathcal{P}_{-}\right) \cdot \mathbf{u} \sqrt{a} d z_{1} d z_{2} \mp \int_{\Sigma_{ \pm}} V p^{0} \sqrt{a} d z_{1} d z_{2} .
\end{aligned}
$$

Equality (3.24) implies uniqueness of solutions to problem (3.18)-(3.20). Concerning existence, equality (3.24) allows to obtain the uniform bounds for $\gamma(\mathbf{u})$ in $L^{\infty}\left(0, T ; \mathcal{V}_{M}(\omega)\right)$, for $p^{0}$ in $L^{\infty}\left(0, T ; L^{2}(\Omega)\right)$ and for $\partial_{z_{3}} p^{0}$ in $L^{2}\left(0, T ; L^{2}(\Omega)\right)$. Using Lemma 6 and the classical weak compactness reasoning, we conclude the existence of at least one solution.

Remark 8. Note that the equation (3.23) can be used to decouple the problem. Thus we first can solve the membrane problem with slightly changed coefficients and with time as a parameter in the equation. In the second step we plug this solution into (3.19). This approach can also lead to alternative existence proof. Namely, standard existence theory for membrane shell model applied on (3.23) yields that there is a unique $\mathbf{u} \in H^{2}\left(0, T ; \mathcal{V}_{M}(\omega)\right)$ solving (3.23). Then the standard parabolic theory for (3.19) implies the existence of $p^{0}$ in $C\left([0, T] ; L^{2}(\Omega)\right)$ and such $\partial_{z_{3}} p^{0}$ in $L^{2}\left(0, T ; L^{2}(\Omega)\right)$. Further regularity is standard.

Standards computations give

$p^{0}-\int_{-1 / 2}^{1 / 2} p^{0} d z_{3}=-V(t) y_{3}+\frac{4 \bar{\beta}}{\pi^{2}} \sum_{m=0}^{\infty} \int_{0}^{t} e^{-\frac{(2 m+1)^{2} \pi^{2}}{\bar{\beta}}(t-s)} \partial_{t} V d s \frac{(-1)^{m}}{(2 m+1)^{2}} \sin \left((2 m+1) \pi y_{3}\right)$,

with $\bar{\beta}=\beta+\frac{\alpha^{2}}{\tilde{\lambda}+2}$. After inserting (3.22) into (3.18) for displacement we get a standard elastic membrane shell equations with modified coefficients. Then we use (3.22) to compute the mean pressure and finally (3.25) to reconstruct the pressure fluctuation.

The main result of the paper is the following theorem.

Theorem 9. Let us suppose Assumption 4. Let $\{\mathbf{u}(\varepsilon), p(\varepsilon)\} \in H^{1}(0, T ; \mathcal{V}(\Omega)) \times H^{1}\left(0, T ; H^{1}(\Omega)\right)$ be the unique solution of (3.16) and let $\left\{\mathbf{u}, p^{0}\right\}$ be the unique solution for (3.18)-(3.20). Then we obtain

$$
\begin{array}{lr}
\mathbf{u}(\varepsilon) \rightarrow \mathbf{u} & \text { strongly in } C\left([0, T] ; H^{1}(\Omega) \times H^{1}(\Omega) \times L^{2}(\Omega)\right), \\
\gamma^{\varepsilon}(\mathbf{u}(\varepsilon)) \rightarrow \gamma^{0} & \text { strongly in } C\left([0, T] ; L^{2}\left(\Omega ; \mathbb{R}^{3 \times 3}\right)\right), \\
p(\varepsilon) \rightarrow p^{0} & \text { strongly in } C\left([0, T] ; L^{2}(\Omega)\right), \\
\frac{\partial p(\varepsilon)}{\partial z_{3}} \rightarrow \frac{\partial p^{0}}{\partial z_{3}} & \text { strongly in } L^{2}\left(0, T ; L^{2}(\Omega)\right),
\end{array}
$$


where

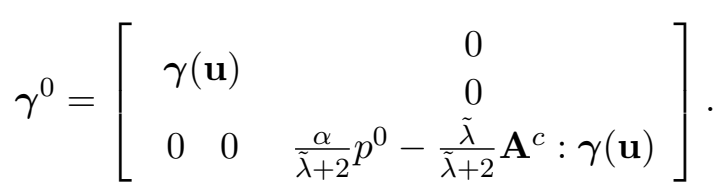

Remark 10. We observe differences between convergence theorem (Theorem 6) from [22] and this result. The most notable difference is in the structure of $\gamma^{0}$.

As a consequence of the convergence of the term $\boldsymbol{\gamma}^{\varepsilon}(\mathbf{u}(\varepsilon))$, we obtain the convergence of the scaled stress tensor.

Corollary 11. For the stress tensor $\sigma(\varepsilon)=\mathcal{C}\left(\mathbf{Q}(\varepsilon) \boldsymbol{\gamma}^{\varepsilon}(\mathbf{u}(\varepsilon)) \mathbf{Q}(\varepsilon)^{T}\right)-\alpha p(\varepsilon) \mathbf{I}$ one has

$$
\frac{1}{\varepsilon} \sigma(\varepsilon) \rightarrow \sigma=\mathcal{C}\left(\mathbf{Q} \gamma^{0} \mathbf{Q}^{T}\right)-\alpha p^{0} \mathbf{I} \quad \text { strongly in } C\left([0, T] ; L^{2}\left(\Omega ; \mathbb{R}^{3 \times 3}\right)\right) \text {. }
$$

The limit stress in the local contravariant basis $\mathbf{Q}=\left(\mathbf{a}^{1} \mathbf{a}^{2} \mathbf{a}^{3}\right)$ is given by

$$
\mathbf{Q}^{T} \sigma \mathbf{Q}=\left[\begin{array}{cc}
\left.-\frac{2 \alpha}{\tilde{\lambda}+2} p^{0} \mathbf{I}+\frac{2 \tilde{\lambda}}{\tilde{\lambda}+2}\left(\mathbf{A}^{c}: \boldsymbol{\gamma}(\mathbf{u})\right) \mathbf{I}+2 \mathbf{A}^{c} \boldsymbol{\gamma}(\mathbf{u})\right) \mathbf{A}^{c} & 0 \\
0 & 0
\end{array}\right]
$$

\section{A priori estimates}

Fundamental for a priori estimates for elliptic membrane shells (clamped at all lateral surface and elliptic) is the following three-dimensional inequality of Korn's type for a family of linearly elastic elliptic membrane shells.

Theorem 12 ([5, Theorem 4.3-1], [7, Theorem 4.1]). Assume that $\mathbf{X} \in C^{3}\left(\bar{\omega} ; \mathbb{R}^{3}\right)$ parameterizes an elliptic surface. Then there exist constants $\varepsilon_{0}>0, C>0$ such that for all $\varepsilon \in\left(0, \varepsilon_{0}\right)$ one has

$$
\left\|v_{1}\right\|_{H^{1}(\Omega)}^{2}+\left\|v_{2}\right\|_{H^{1}(\Omega)}^{2}+\left\|v_{3}\right\|_{L^{2}(\Omega)}^{2} \leq C\left\|\gamma^{\varepsilon}(\mathbf{v})\right\|_{L^{2}\left(\Omega ; \mathbb{R}^{3 \times 3}\right)}^{2}, \quad \mathbf{v} \in \mathcal{V}(\Omega) .
$$

Remark 13. Note that the above estimate applies to the functions on the three-dimensional domain $\Omega$ and will be the basis for the a priori estimates for the solution of (3.16), while in Lemma 6 the estimate was for functions on $\omega$ ad is the basis for the existence and uniqueness of the solution of the limit model (3.18)-(3.20).

Next we state the asymptotic properties of the coefficients in the equation (3.16). Direct calculation shows that there are constants $m_{g}, M_{g}$, independent of $\varepsilon \in\left(0, \varepsilon_{0}\right)$, such that for all $z \in \Omega$,

$$
m_{g} \leq \sqrt{g(\varepsilon)} \leq M_{g}
$$

The functions $\mathbf{g}^{i}(\varepsilon), \mathbf{g}_{i}(\varepsilon), g^{i j}(\varepsilon), g(\varepsilon), \Gamma_{j k}^{i}(\varepsilon), \mathbf{Q}(\varepsilon)$ are in $C(\bar{\Omega})$ by assumptions. Moreover, there is a constant $C>0$ such that for all $\varepsilon \in\left(0, \varepsilon_{0}\right)$,

$$
\begin{aligned}
& \left\|\mathbf{g}^{i}(\varepsilon)-\mathbf{a}^{i}\right\|_{\infty}+\left\|\mathbf{g}_{i}(\varepsilon)-\mathbf{a}_{i}\right\|_{\infty} \leq C \varepsilon \\
& \left\|\frac{\partial}{\partial z_{3}} \sqrt{g(\varepsilon)}\right\|_{\infty}+\|\sqrt{g(\varepsilon)}-\sqrt{a}\|_{\infty} \leq C \varepsilon \\
& \|\mathbf{Q}(\varepsilon)-\mathbf{Q}\|_{\infty} \leq C \varepsilon \\
& \left\|\Gamma_{j k}^{i}(\varepsilon)-\Gamma_{j k}^{i}(0)\right\|_{\infty} \leq C \varepsilon
\end{aligned}
$$


where $\|\cdot\|_{\infty}$ is the norm in $C(\bar{\Omega})$. For proof see $[7,8]$. In addition, in [5, Theorem 3.3-1] and [8, Lemma 3.1] the asymptotic of the Christoffel symbols is given by

$$
\Gamma^{\kappa}(\varepsilon)=\left[\begin{array}{ccc}
\Gamma_{11}^{\kappa} & \Gamma_{12}^{\kappa} & -b_{1}^{\kappa} \\
\Gamma_{21}^{\kappa} & \Gamma_{22}^{\kappa} & -b_{2}^{\kappa} \\
-b_{1}^{\kappa} & -b_{2}^{\kappa} & 0
\end{array}\right]+O(\varepsilon)
$$

where $\kappa=1,2$ and

$$
\boldsymbol{\Gamma}^{3}(\varepsilon)=\left[\begin{array}{ccc}
b_{11} & b_{12} & 0 \\
b_{21} & b_{22} & 0 \\
0 & 0 & 0
\end{array}\right]+O(\varepsilon) .
$$

In the following two lemmas we derive the a priori estimates in a classical way. The estimates are similar, but different from the flexural case. Namely, the scaling of $\boldsymbol{\gamma}^{\varepsilon}(\mathbf{u}(\varepsilon))$ is different.

Lemma 14. There is $C>0$ and $\varepsilon_{0}>0$ such that for all $\varepsilon \in\left(0, \varepsilon_{0}\right)$ one has

$$
\left\|\gamma^{\varepsilon}(\mathbf{u}(\varepsilon))\right\|_{L^{\infty}\left(0, T ; L^{2}\left(\Omega ; \mathbb{R}^{3 \times 3}\right)\right)},\|p(\varepsilon)\|_{L^{\infty}\left(0, T ; L^{2}(\Omega ; \mathbb{R})\right)},\left\|\varepsilon \nabla^{\varepsilon} p(\varepsilon)\right\|_{L^{2}\left(0, T ; L^{2}\left(\Omega ; \mathbb{R}^{3}\right)\right)} \leq C .
$$

Proof. We set $\mathbf{v}=\frac{\partial \mathbf{u}(\varepsilon)}{\partial t}$ and $q=p(\varepsilon)$ in (3.16) and sum up the equations. After noticing that the pressure term from the first equation cancels with the compression term from the second equation we obtain

$$
\begin{aligned}
\frac{1}{2} \varepsilon & \frac{d}{d t} \int_{\Omega} \mathcal{C}\left(\mathbf{Q}(\varepsilon) \boldsymbol{\gamma}^{\varepsilon}(\mathbf{u}(\varepsilon)) \mathbf{Q}(\varepsilon)^{T}\right):\left(\mathbf{Q}(\varepsilon) \boldsymbol{\gamma}^{\varepsilon}(\mathbf{u}(\varepsilon)) \mathbf{Q}(\varepsilon)^{T}\right) \sqrt{g(\varepsilon)} d z \\
& +\frac{1}{2} \beta \varepsilon \frac{d}{d t} \int_{\Omega} p(\varepsilon)^{2} \sqrt{g(\varepsilon)} d z+\varepsilon^{3} \int_{\Omega} \mathbf{Q}(\varepsilon) \nabla^{\varepsilon} p(\varepsilon) \cdot \mathbf{Q}(\varepsilon) \nabla^{\varepsilon} p(\varepsilon) \sqrt{g(\varepsilon)} d z \\
= & \varepsilon \int_{\Sigma_{ \pm}} \mathcal{P}_{ \pm} \cdot \frac{\partial \mathbf{u}(\varepsilon)}{\partial t} \sqrt{g(\varepsilon)} d s \mp \varepsilon \int_{\Sigma_{ \pm}} V p(\varepsilon) \sqrt{g(\varepsilon)} d s .
\end{aligned}
$$

Dividing the equation by $\varepsilon$ and using the product rule for derivatives with respect to time on the right hand side yield

$$
\begin{aligned}
& \frac{1}{2} \frac{d}{d t}\left(\int_{\Omega} \mathcal{C}\left(\mathbf{Q}(\varepsilon) \boldsymbol{\gamma}^{\varepsilon}(\mathbf{u}(\varepsilon)) \mathbf{Q}(\varepsilon)^{T}\right):\left(\mathbf{Q}(\varepsilon) \boldsymbol{\gamma}^{\varepsilon}(\mathbf{u}(\varepsilon)) \mathbf{Q}(\varepsilon)^{T}\right) \sqrt{g(\varepsilon)} d z+\beta \int_{\Omega} p(\varepsilon)^{2} \sqrt{g(\varepsilon)} d z\right) \\
& \quad+\varepsilon^{2} \int_{\Omega} \mathbf{Q}(\varepsilon) \nabla^{\varepsilon} p(\varepsilon) \cdot \mathbf{Q}(\varepsilon) \nabla^{\varepsilon} p(\varepsilon) \sqrt{g(\varepsilon)} d z \\
& =\frac{d}{d t} \int_{\Sigma_{ \pm}} \mathcal{P}_{ \pm} \cdot \mathbf{u}(\varepsilon) \sqrt{g(\varepsilon)} d s-\int_{\Sigma_{ \pm}} \frac{\partial \mathcal{P}_{ \pm}}{\partial t} \cdot \mathbf{u}(\varepsilon) \sqrt{g(\varepsilon)} d s \mp \int_{\Sigma_{ \pm}} V p(\varepsilon) \sqrt{g(\varepsilon)} d s .
\end{aligned}
$$

Now we use the Newton-Leibnitz formula for the terms on the right hand side and the notation

$$
\mathcal{P}=\left(\mathcal{P}_{+}+\mathcal{P}_{-}\right) z_{3}+\frac{\mathcal{P}_{+}-\mathcal{P}_{-}}{2}, \quad \mathcal{V}=2 V z_{3}
$$

to obtain

$$
\begin{aligned}
& \frac{1}{2} \frac{d}{d t}\left(\int_{\Omega} \mathcal{C}\left(\mathbf{Q}(\varepsilon) \boldsymbol{\gamma}^{\varepsilon}(\mathbf{u}(\varepsilon)) \mathbf{Q}(\varepsilon)^{T}\right):\left(\mathbf{Q}(\varepsilon) \boldsymbol{\gamma}^{\varepsilon}(\mathbf{u}(\varepsilon)) \mathbf{Q}(\varepsilon)^{T}\right) \sqrt{g(\varepsilon)} d z+\beta \int_{\Omega} p(\varepsilon)^{2} \sqrt{g(\varepsilon)} d z\right) \\
&+\varepsilon^{2} \int_{\Omega} \mathbf{Q}(\varepsilon) \nabla^{\varepsilon} p(\varepsilon) \cdot \mathbf{Q}(\varepsilon) \nabla^{\varepsilon} p(\varepsilon) \sqrt{g(\varepsilon)} d z \\
&= \frac{d}{d t} \int_{\Omega} \frac{\partial}{\partial z_{3}}(\mathcal{P} \cdot \mathbf{u}(\varepsilon) \sqrt{g(\varepsilon)}) d z-\int_{\Omega} \frac{\partial}{\partial z_{3}}\left(\frac{\partial \mathcal{P}}{\partial t} \cdot \mathbf{u}(\varepsilon) \sqrt{g(\varepsilon)}\right) d z \\
& \quad-\int_{\Omega} \frac{\partial}{\partial z_{3}}(\mathcal{V} p(\varepsilon) \sqrt{g(\varepsilon)}) d z .
\end{aligned}
$$


Next we integrate this inequality over time

$$
\begin{aligned}
& \frac{1}{2} \int_{\Omega} \mathcal{C}\left(\mathbf{Q}(\varepsilon) \boldsymbol{\gamma}^{\varepsilon}(\mathbf{u}(\varepsilon)) \mathbf{Q}(\varepsilon)^{T}\right):\left(\mathbf{Q}(\varepsilon) \boldsymbol{\gamma}^{\varepsilon}(\mathbf{u}(\varepsilon)) \mathbf{Q}(\varepsilon)^{T}\right) \sqrt{g(\varepsilon)} d z+\frac{1}{2} \beta \int_{\Omega} p(\varepsilon)^{2} \sqrt{g(\varepsilon)} d z \\
& \quad+\varepsilon^{2} \int_{0}^{t} \int_{\Omega} \mathbf{Q}(\varepsilon) \nabla^{\varepsilon} p(\varepsilon) \cdot \mathbf{Q}(\varepsilon) \nabla^{\varepsilon} p(\varepsilon) \sqrt{g(\varepsilon)} d z d \tau \\
&=\frac{1}{2}\left(\int_{\Omega} \mathcal{C}\left(\mathbf{Q}(\varepsilon) \boldsymbol{\gamma}^{\varepsilon}\left(\left.\mathbf{u}(\varepsilon)\right|_{t=0}\right) \mathbf{Q}(\varepsilon)^{T}\right):\left(\mathbf{Q}(\varepsilon) \boldsymbol{\gamma}^{\varepsilon}\left(\left.\mathbf{u}(\varepsilon)\right|_{t=0}\right) \mathbf{Q}(\varepsilon)^{T}\right) \sqrt{g(\varepsilon)} d z+\left.\beta \int_{\Omega} p(\varepsilon)^{2}\right|_{t=0} \sqrt{g(\varepsilon)} d z\right) \\
& \quad+\int_{\Omega} \frac{\partial}{\partial z_{3}}(\mathcal{P} \cdot \mathbf{u}(\varepsilon) \sqrt{g(\varepsilon)}) d z-\int_{\Omega} \frac{\partial}{\partial z_{3}}\left(\left.\left.\mathcal{P}\right|_{t=0} \cdot \mathbf{u}(\varepsilon)\right|_{t=0} \sqrt{g(\varepsilon)}\right) d z-\int_{0}^{t} \int_{\Omega} \frac{\partial}{\partial z_{3}}\left(\frac{\partial \mathcal{P}}{\partial t} \cdot \mathbf{u}(\varepsilon) \sqrt{g(\varepsilon)}\right) d z d \tau \\
& \quad+\int_{0}^{t} \int_{\Omega} \frac{\partial}{\partial z_{3}}(\mathcal{V} p(\varepsilon) \sqrt{g(\varepsilon)}) d z d \tau
\end{aligned}
$$

Since we have sufficient time regularity for $\mathbf{u}(\varepsilon)$, we consider (3.16) for $t=0$. Then $\left.\mathbf{u}(\varepsilon)\right|_{t=0}$ satisfies: for all $\mathbf{v} \in \mathcal{V}(\Omega)$

$$
\begin{aligned}
\int_{\Omega} \mathcal{C} & \left(\mathbf{Q}(\varepsilon) \boldsymbol{\gamma}^{\varepsilon}\left(\left.\mathbf{u}(\varepsilon)\right|_{t=0}\right) \mathbf{Q}(\varepsilon)^{T}\right):\left(\mathbf{Q}(\varepsilon) \boldsymbol{\gamma}^{\varepsilon}(\mathbf{v}) \mathbf{Q}(\varepsilon)^{T}\right) \sqrt{g(\varepsilon)} d z \\
& -\left.\alpha \int_{\Omega} p(\varepsilon)\right|_{t=0} \operatorname{tr}\left(\mathbf{Q}(\varepsilon) \boldsymbol{\gamma}^{\varepsilon}(\mathbf{v}) \mathbf{Q}(\varepsilon)^{T}\right) \sqrt{g(\varepsilon)} d z=\left.\int_{\Sigma_{ \pm}} \mathcal{P}_{ \pm}\right|_{t=0} \cdot \mathbf{v} \sqrt{g(\varepsilon)} d s
\end{aligned}
$$

Since the initial condition is $\left.p(\varepsilon)\right|_{t=0}=0$ this equation is a classical 3D equation of shell-like body in curvilinear coordinates rescaled on the canonical domain. Next, $\left.\mathcal{P}_{ \pm}\right|_{t=0}=0$ and the classical theory (see Ciarlet [5]) yields $\left.\mathbf{u}(\varepsilon)\right|_{t=0}=0$. Using Korn's inequality, positivity of $\mathcal{C}$ and uniform positivity of $\mathbf{Q}(\varepsilon)^{T} \mathbf{Q}(\varepsilon)$ and $g(\varepsilon)$ in (4.5) yields the estimate

$$
\begin{aligned}
& \frac{1}{2} \int_{\Omega} \mathcal{C}\left(\mathbf{Q}(\varepsilon) \boldsymbol{\gamma}^{\varepsilon}(\mathbf{u}(\varepsilon)) \mathbf{Q}(\varepsilon)^{T}\right):\left(\mathbf{Q}(\varepsilon) \boldsymbol{\gamma}^{\varepsilon}(\mathbf{u}(\varepsilon)) \mathbf{Q}(\varepsilon)^{T}\right) \sqrt{g(\varepsilon)} d z+\frac{1}{2} \beta \int_{\Omega} p(\varepsilon)^{2} \sqrt{g(\varepsilon)} d z \\
& \quad+\varepsilon^{2} \int_{0}^{t} \int_{\Omega} \mathbf{Q}(\varepsilon) \nabla^{\varepsilon} p(\varepsilon) \cdot \mathbf{Q}(\varepsilon) \nabla^{\varepsilon} p(\varepsilon) \sqrt{g(\varepsilon)} d z d \tau \leq C
\end{aligned}
$$

Since $\mathcal{C}$ is positive definite and since $g(\varepsilon)$ is uniformly positive definite (see [5, Theorem 3.3-1]) we obtain the following uniform bounds

$$
\left\|\mathbf{Q}(\varepsilon) \boldsymbol{\gamma}^{\varepsilon}(\mathbf{u}(\varepsilon)) \mathbf{Q}(\varepsilon)^{T}\right\|_{L^{\infty}\left(0, T ; L^{2}\left(\Omega ; \mathbb{R}^{3 \times 3}\right)\right)}, \quad\|p(\varepsilon)\|_{L^{\infty}\left(0, T ; L^{2}(\Omega)\right)}, \quad\left\|\varepsilon \mathbf{Q}(\varepsilon) \nabla^{\varepsilon} p(\varepsilon)\right\|_{L^{2}\left(0, T ; L^{2}\left(\Omega ; \mathbb{R}^{3}\right)\right)} .
$$

Since $\mathbf{Q}(\varepsilon)^{T} \mathbf{Q}(\varepsilon)$ is uniformly positive definite these estimates imply uniform bounds for

$$
\left\|\boldsymbol{\gamma}^{\varepsilon}(\mathbf{u}(\varepsilon)) \mathbf{Q}(\varepsilon)^{T}\right\|_{L^{\infty}\left(0, T ; L^{2}\left(\Omega ; \mathbb{R}^{3 \times 3}\right)\right)}, \quad\|p(\varepsilon)\|_{L^{\infty}\left(0, T ; L^{2}(\Omega)\right)}, \quad\left\|\varepsilon \nabla^{\varepsilon} p(\varepsilon)\right\|_{L^{2}\left(0, T ; L^{2}\left(\Omega ; \mathbb{R}^{3}\right)\right)} .
$$

Applying the uniform bounds for $\mathbf{Q}(\varepsilon)^{T} \mathbf{Q}(\varepsilon)$ once again implies the statement of the lemma.

We now first take the time derivative of the first equation in (3.16) and then insert $\mathbf{v}=\frac{\partial \mathbf{u}(\varepsilon)}{\partial t}$ as a test functions. Then we take $q=\frac{\partial p(\varepsilon)}{\partial t}$ as test functions in the second equation in (3.16) and sum the equations. We obtain

$$
\begin{aligned}
& \int_{\Omega} \mathcal{C}\left(\mathbf{Q}(\varepsilon) \boldsymbol{\gamma}^{\varepsilon}\left(\frac{\partial \mathbf{u}(\varepsilon)}{\partial t}\right) \mathbf{Q}(\varepsilon)^{T}\right):\left(\mathbf{Q}(\varepsilon) \boldsymbol{\gamma}^{\varepsilon}\left(\frac{\partial \mathbf{u}(\varepsilon)}{\partial t}\right) \mathbf{Q}(\varepsilon)^{T}\right) \sqrt{g(\varepsilon)} d z \\
& \quad+\beta \int_{\Omega} \frac{\partial p(\varepsilon)}{\partial t} \frac{\partial p(\varepsilon)}{\partial t} \sqrt{g(\varepsilon)} d z+\frac{1}{2} \varepsilon^{2} \frac{d}{d t} \int_{\Omega} \mathbf{Q}(\varepsilon) \nabla^{\varepsilon} p(\varepsilon) \cdot \mathbf{Q}(\varepsilon) \nabla^{\varepsilon} p(\varepsilon) \sqrt{g(\varepsilon)} d z \\
& =\int_{\Sigma_{ \pm}} \frac{\partial \mathcal{P}_{ \pm}}{\partial t} \cdot \frac{\partial \mathbf{u}(\varepsilon)}{\partial t} \sqrt{g(\varepsilon)} d s \mp \int_{\Sigma_{ \pm}} V \frac{\partial p(\varepsilon)}{\partial t} \sqrt{g(\varepsilon)} d s
\end{aligned}
$$

Similarly as in Lemma 14 from this equality we obtain 
Lemma 15. There is $C>0$ and $\varepsilon_{0}>0$ such that for all $\varepsilon \in\left(0, \varepsilon_{0}\right)$ one has

$$
\left\|\boldsymbol{\gamma}^{\varepsilon}\left(\frac{\partial \mathbf{u}(\varepsilon)}{\partial t}\right)\right\|_{L^{2}\left(0, T ; L^{2}\left(\Omega ; \mathbb{R}^{3 \times 3}\right)\right)},\left\|\frac{\partial p(\varepsilon)}{\partial t}\right\|_{L^{2}\left(0, T ; L^{2}(\Omega ; \mathbb{R})\right)},\left\|\varepsilon \nabla^{\varepsilon} p(\varepsilon)\right\|_{L^{\infty}\left(0, T ; L^{2}\left(\Omega ; \mathbb{R}^{3}\right)\right)} \leq C .
$$

As a consequence of the scaled Korn's inequality from Theorem 12 we obtain

Corollary 16. Let us suppose Assumption 4 and let $\{\mathbf{u}(\varepsilon), p(\varepsilon)\}$ be the solution for problem (3.16). Then there is $C>0$ and $\varepsilon_{0}>0$ such that for all $\varepsilon \in\left(0, \varepsilon_{0}\right)$ one has

$$
\begin{gathered}
\left\|\boldsymbol{\gamma}^{\varepsilon}(\mathbf{u}(\varepsilon))\right\|_{H^{1}\left(0, T ; L^{2}\left(\Omega ; \mathbb{R}^{9}\right)\right)},\left\|u_{1}(\varepsilon)\right\|_{H^{1}\left(0, T ; H^{1}(\Omega)\right)},\left\|u_{2}(\varepsilon)\right\|_{H^{1}\left(0, T ; H^{1}(\Omega)\right)},\left\|u_{3}(\varepsilon)\right\|_{H^{1}\left(0, T ; L^{2}(\Omega)\right)}, \\
\|p(\varepsilon)\|_{H^{1}\left(0, T ; L^{2}(\Omega ; \mathbb{R})\right)},\left\|\frac{\partial p(\varepsilon)}{\partial z_{3}}\right\|_{L^{\infty}\left(0, T ; L^{2}(\Omega ; \mathbb{R})\right)} \leq C .
\end{gathered}
$$

In addition, there are $u_{1}, u_{2} \in H^{1}\left(0, T ; H^{1}\left(\Omega ; \mathbb{R}^{3}\right)\right)$, $u_{3} \in H^{1}\left(0, T ; L^{2}\left(\Omega ; \mathbb{R}^{3}\right)\right), p^{0} \in H^{1}\left(0, T ; L^{2}(\Omega ; \mathbb{R})\right)$ and $\gamma^{0} \in L^{\infty}\left(0, T ; L^{2}\left(\Omega ; \mathbb{R}^{3 \times 3}\right)\right)$ such that on a subsequence one has

$$
\begin{aligned}
& u_{j}(\varepsilon) \rightarrow u_{j} \text { weakly in } H^{1}\left(0, T ; H^{1}(\Omega)\right), \quad j=1,2, \\
& u_{3}(\varepsilon) \rightarrow u_{3} \text { weakly in } H^{1}\left(0, T ; L^{2}(\Omega)\right), \\
& p(\varepsilon) \rightarrow p^{0} \text { weakly in } H^{1}\left(0, T ; L^{2}(\Omega ; \mathbb{R})\right), \\
& \frac{\partial p(\varepsilon)}{\partial z_{3}} \rightarrow \frac{\partial p^{0}}{\partial z_{3}} \text { weakly in } L^{2}\left(0, T ; L^{2}(\Omega ; \mathbb{R})\right) \quad \text { and weak *in } \quad L^{\infty}\left(0, T ; L^{2}(\Omega ; \mathbb{R})\right), \\
& \boldsymbol{\gamma}^{\varepsilon}(\mathbf{u}(\varepsilon)) \rightarrow \gamma^{0} \text { weakly in } H^{1}\left(0, T ; L^{2}\left(\Omega ; \mathbb{R}^{3 \times 3}\right)\right) .
\end{aligned}
$$

Proof. Straightforward.

Since $\boldsymbol{\gamma}^{\varepsilon}(\mathbf{u}(\varepsilon))$ depends on $\mathbf{u}(\varepsilon)$ one expects that the limits $\mathbf{u}=\left(u_{1}, u_{2}, u_{3}\right)$ and $\boldsymbol{\gamma}^{0}$ are related. The following theorem gives the precise relationship. The following theorem is fundamental for obtaining the limit model in classical elliptic membrane shell derivation as well as in the present derivation. Its proof is a collection of particular statements in the proof of Theorem 4.4-1 in [5]. Therefore we just sketch it here.

Theorem 17. For any $\mathbf{v} \in \mathcal{V}(\Omega)$ let $\boldsymbol{\gamma}^{\varepsilon}(\mathbf{v})$ be given by (3.17) and let the tensor $\boldsymbol{\gamma}(\mathbf{v})$ be given by (2.5). Let the family $(\mathbf{w}(\varepsilon))_{\varepsilon>0} \subset \mathcal{V}(\Omega)$ satisfies

$$
\begin{aligned}
& w_{j}(\varepsilon) \rightarrow w_{j} \text { weakly in } H^{1}(\Omega), \quad j=1,2, \\
& w_{3}(\varepsilon) \rightarrow w_{3} \text { weakly in } L^{2}(\Omega), \\
& \boldsymbol{\gamma}^{\varepsilon}(\mathbf{w}(\varepsilon)) \rightarrow \tilde{\boldsymbol{\gamma}}^{0} \text { weakly in } L^{2}\left(\Omega ; \mathbb{R}^{3 \times 3}\right)
\end{aligned}
$$

as $\varepsilon \rightarrow 0$. Then $\mathbf{w}=\left(w_{1}, w_{2}, w_{3}\right)$ is independent of transverse variable $z_{3}$, belongs to $\mathcal{V}_{M}(\omega)=$ $H_{0}^{1}(\omega) \times H_{0}^{1}(\omega) \times L^{2}(\omega)$, and satisfies

$$
\tilde{\gamma}_{\alpha \beta}^{0}=\gamma_{\alpha \beta}(\mathbf{w}), \quad \alpha, \beta \in\{1,2\} .
$$

Proof. From $\boldsymbol{\gamma}^{\varepsilon}(\mathbf{w}(\varepsilon)) \rightarrow \tilde{\boldsymbol{\gamma}}^{0}$ we obtain that

$$
\varepsilon \boldsymbol{\gamma}^{\varepsilon}(\mathbf{w}(\varepsilon))=\boldsymbol{\gamma}_{z}(\mathbf{w}(\varepsilon))+\varepsilon \boldsymbol{\gamma}_{y}(\mathbf{w}(\varepsilon))-\varepsilon \sum_{i=1}^{3} w_{i}(\varepsilon) \boldsymbol{\Gamma}^{i}(\varepsilon) \rightarrow 0
$$

strongly in $L^{2}\left(\Omega ; \mathbb{R}^{3}\right)$. From the convergences in (4.8) and asymptotics of $\boldsymbol{\Gamma}^{i}(\varepsilon)$ given in (4.3) and (4.4) we have that

$$
\varepsilon \sum_{i=1}^{3} w_{i}(\varepsilon) \boldsymbol{\Gamma}^{i}(\varepsilon) \rightarrow 0
$$


strongly in $L^{2}\left(\Omega ; \mathbb{R}^{3 \times 3}\right)$. Also (4.8) implies that $\varepsilon \boldsymbol{\gamma}_{y}(\mathbf{w}(\varepsilon)) \rightarrow 0$ strongly in $H^{-1}\left(\Omega ; \mathbb{R}^{3 \times 3}\right)$. Finally $\gamma_{z}(\mathbf{w}(\varepsilon)) \rightarrow 0$ strongly in $L^{2}\left(\omega ; H^{-1}\left(-1 / 2,1 / 2 ; \mathbb{R}^{3 \times 3}\right)\right)$ and weakly in $L^{2}\left(\Omega ; \mathbb{R}^{3 \times 3}\right)$. From the definition of $\boldsymbol{\gamma}_{z}$ in (3.17) we obtain that $\partial_{3} w_{i}(\varepsilon) \rightarrow 0$ strongly in $H^{-1}(\Omega)$. Therefore $\mathbf{w}$ is independent of the transverse variable $z_{3}$. Then it is straightforward to conclude that $\mathbf{w} \in \mathcal{V}_{M}(\omega)$.

Now, the convergences $\gamma_{\alpha \beta}^{\varepsilon}(\mathbf{w}(\varepsilon)) \rightarrow \tilde{\gamma}_{\alpha \beta}^{0}, \alpha, \beta \in\{1,2\}$ from (4.8), using the definition of $\boldsymbol{\gamma}^{\varepsilon}$ from (3.17), imply

$$
\tilde{\gamma}_{\alpha \beta}^{0}=\lim _{\varepsilon \rightarrow 0}\left(\frac{1}{2}\left(\partial_{\alpha} w_{\beta}(\varepsilon)+\partial_{\beta} w_{\alpha}(\varepsilon)\right)-\sum_{i=1}^{3} w_{i}(\varepsilon) \Gamma_{\alpha \beta}^{i}(\varepsilon)\right) .
$$

Using the asymptotics of $\boldsymbol{\Gamma}^{i}(\varepsilon)$ from (4.3) and (4.4) together with the remaining convergences in (4.8) yield

$$
\tilde{\gamma}_{\alpha \beta}^{0}=\frac{1}{2}\left(\partial_{\alpha} w_{\beta}+\partial_{\beta} w_{\alpha}\right)-\sum_{i=1}^{3} w_{i} \Gamma_{\alpha \beta}^{i}(0)=\gamma_{\alpha \beta}(\mathbf{w}) .
$$

Remark 18. In order to apply Theorem 17 we need pointwise convergences for every $t \in[0, T]$. The estimates from Corollary 16 (i.e., Lemma 14 and Lemma 15) imply that we are in the same position as in Remark 14 from [22] for $u_{1}(\varepsilon), u_{2}(\varepsilon), p(\varepsilon)$ and $\boldsymbol{\gamma}^{\varepsilon}(\boldsymbol{u}(\varepsilon))$, i.e.

$$
\begin{aligned}
& u_{\alpha}(\varepsilon)(t) \rightarrow u_{\alpha}(t) \text { weakly in } H^{1}(\Omega) \quad \text { for every } t \in[0, T], \quad \alpha \in\{1,2\}, \\
& p(\varepsilon)(t) \rightarrow p^{0}(t) \text { weakly in } L^{2}(\Omega), \\
& \boldsymbol{\gamma}^{\varepsilon}(\mathbf{u}(\varepsilon))(t) \rightarrow \boldsymbol{\gamma}^{0}(t) \text { weakly in } L^{2}\left(\Omega ; \mathbb{R}^{3 \times 3}\right),
\end{aligned}
$$

for every $t \in[0, T]$.

In the case of $u_{3}(\varepsilon)$ we argue similarly. Corollary 16 implies that $u_{3}(\varepsilon)$ is uniformly bounded in $C^{0,1 / 2}\left([0, T], L^{2}(\Omega)\right)$. Therefore by the Aubin-Lions lemma (see [29]), there is a subsequence such that the $\left\{u_{3}(\varepsilon)\right\}$ converges to $u_{3}$ also in $C\left([0, T] ; H^{-1}(\Omega)\right)$. Let $\varphi \in L^{2}(\Omega)$. Then for every $\delta>0$, there exists $\varphi_{\delta} \in C_{0}^{\infty}(\Omega)$ such that $\left\|\varphi-\varphi_{\delta}\right\|_{L^{2}(\Omega)} \leq \delta$. Then

$$
\begin{aligned}
& \sup _{0 \leq t \leq T}\left|\int_{\Omega}\left(u_{3}(\varepsilon)(t)-u_{3}(t)\right) \varphi d x\right| \\
& \quad \leq \sup _{0 \leq t \leq T}\left|\int_{\Omega}\left(u_{3}(\varepsilon)(t)-u_{3}(t)\right)\left(\varphi-\varphi_{\delta}\right) d x\right|+\sup _{0 \leq t \leq T}\left|\int_{\Omega} \varphi_{\delta}\left(u_{3}(\varepsilon)(t)-u_{3}(t)\right) d x\right| \\
& \quad \leq \delta\left\|u_{3}(\varepsilon)-u_{3}\right\|_{C\left([0, T] ; L^{2}\left(\Omega ; \mathbb{R}^{3}\right)\right)}+\left\|\varphi_{\delta}\right\|_{H^{1}(\Omega)}\left\|u_{3}(\varepsilon)-u_{3}\right\|_{C\left([0, T] ; H^{-1}\left(\Omega ; \mathbb{R}^{3}\right)\right)} \leq \bar{C} \delta
\end{aligned}
$$

for $\varepsilon \leq \varepsilon_{0}(\delta)$. Therefore

$$
\lim _{\varepsilon \rightarrow 0} \sup _{0 \leq t \leq T}\left|\int_{\Omega}\left(u_{3}(\varepsilon)(t)-u_{3}(t)\right) \varphi d x\right| \leq \bar{C} \delta
$$

which yields

$$
u_{3}(\varepsilon)(t) \rightarrow u_{3}(t) \text { weakly in } L^{2}\left(\Omega ; \mathbb{R}^{3}\right) \quad \text { for every } t \in[0, T] .
$$

Thus we may apply Theorem 17 , with $\mathbf{w}(\varepsilon)=\mathbf{u}(\varepsilon)(t)$, for each $t \in[0, T]$ and conclude that the limit points of $\{\mathbf{u}(\varepsilon)(t)\}$ belong to $\mathcal{V}_{M}(\omega)$.

Moreover we conclude that

$$
\gamma_{\alpha \beta}^{0}=\gamma_{\alpha \beta}(\mathbf{u}), \quad \alpha, \beta \in\{1,2\}
$$




\section{Derivation of the limit model}

In this section we derive the limit model in two steps by taking the limit in (3.16) for two choices of test functions. Then in the Step 3 we prove the strong convergence of the strain and pressure. Finally in the Step 4 we prove the strong convergence of displacements.

SteP 1 (Identification of $\gamma_{i 3}^{0}$ ).We take the limit as $\varepsilon \rightarrow 0$ in the first equation in (3.16) divided by $\varepsilon$ and obtain

$$
\begin{aligned}
\int_{\Omega} \mathcal{C}\left(\mathbf{Q}(0) \boldsymbol{\gamma}^{0} \mathbf{Q}(0)^{T}\right):\left(\mathbf{Q}(0) \boldsymbol{\gamma}_{z}(\mathbf{v}) \mathbf{Q}(0)^{T}\right) \sqrt{g(0)} d z-\alpha \int_{\Omega} p^{0} \operatorname{tr}\left(\mathbf{Q}(0) \boldsymbol{\gamma}_{z}(\mathbf{v}) \mathbf{Q}(0)^{T}\right) \sqrt{g(0)} d z=0, \\
\mathbf{v} \in \mathcal{V}(\Omega), \text { a.e. } t \in[0, T] .
\end{aligned}
$$

Using $\mathbf{Q}(0)=\mathbf{Q}$ and $g(0)=a$, and the definition of $\gamma_{z}$ and the function space $\mathcal{V}(\Omega)$ yield

$$
\left(\mathbf{Q}^{T}\left(\mathcal{C}\left(\mathbf{Q} \gamma^{0} \mathbf{Q}^{T}\right)-\alpha p^{0} \mathbf{I}\right) \mathbf{Q}\right)_{i 3}=0, \quad i=1,2,3 .
$$

This implies

$$
\left(\left(\tilde{\lambda} \operatorname{tr}\left(\mathbf{Q} \boldsymbol{\gamma}^{0} \mathbf{Q}^{T}\right)-\alpha p^{0}\right) \mathbf{Q}^{T} \mathbf{Q}+2 \mathbf{Q}^{T} \mathbf{Q} \boldsymbol{\gamma}^{0} \mathbf{Q}^{T} \mathbf{Q}\right)_{i 3}=0, \quad i=1,2,3 .
$$

Since

$$
\mathbf{Q}^{T} \mathbf{Q}=\left[\begin{array}{cc}
\mathbf{A}^{c} & 0 \\
0 & 1
\end{array}\right]
$$

we obtain expressions of the third column of $\gamma^{0}$ in terms of the rest of elements

$$
\left(\mathbf{Q}^{T} \mathbf{Q} \boldsymbol{\gamma}^{0}\right)_{13}=\left(\mathbf{Q}^{T} \mathbf{Q} \boldsymbol{\gamma}^{0}\right)_{23}=\tilde{\lambda} \operatorname{tr}\left(\mathbf{Q}^{T} \mathbf{Q} \boldsymbol{\gamma}^{0}\right)-\alpha p^{0}+2 \boldsymbol{\gamma}_{33}^{0}=0
$$

The first two equations imply that

$$
\mathbf{A}^{c}\left[\begin{array}{c}
\gamma_{13}^{0} \\
\gamma_{23}^{0}
\end{array}\right]=0
$$

and since $\mathbf{A}^{c}$ is positive definite we obtain that $\gamma_{13}^{0}=\gamma_{31}^{0}=\gamma_{23}^{0}=\gamma_{32}^{0}=0$. From the third equation in (5.2) we get

$$
\tilde{\lambda} \mathbf{A}^{c}:\left[\begin{array}{cc}
\gamma_{11}^{0} & \gamma_{12}^{0} \\
\gamma_{12}^{0} & \gamma_{22}^{0}
\end{array}\right]-\alpha p^{0}+(\tilde{\lambda}+2) \gamma_{33}^{0}=0
$$

Thus we have obtained the following result. Up to here we followed pages 382-383 from [22].

Lemma 19.

$$
\gamma_{13}^{0}=\gamma_{31}^{0}=\gamma_{23}^{0}=\gamma_{32}^{0}=0, \quad \gamma_{33}^{0}=\frac{\alpha}{\tilde{\lambda}+2} p^{0}-\frac{\tilde{\lambda}}{\tilde{\lambda}+2} \mathbf{A}^{c}: \gamma(\mathbf{u}) .
$$

From this lemma and Theorem 17 we have that $\gamma^{0}$ is of the following form

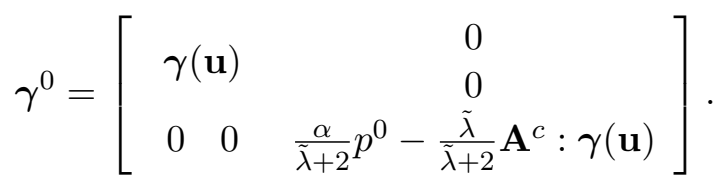

STEP 2 (Taking the second limit). Now we take the limit in (3.16), after division of both equations by $\varepsilon$, for test functions independent of the transversal variable $z_{3}$, i.e., $\mathbf{v} \in H_{0}^{1}\left(\omega ; \mathbb{R}^{3}\right)$, such that

$$
\gamma_{z}(\mathbf{v})=0
$$


Thus $\boldsymbol{\gamma}^{\varepsilon}(\mathbf{v})=\gamma_{y}(\mathbf{v})-\sum_{i=1}^{3} v_{i} \boldsymbol{\Gamma}^{i}(0)$. The equations are

$$
\begin{aligned}
& \int_{\Omega} \mathcal{C}\left(\mathbf{Q}(\varepsilon) \boldsymbol{\gamma}^{\varepsilon}(\mathbf{u}(\varepsilon)) \mathbf{Q}(\varepsilon)^{T}\right):\left(\mathbf{Q}(\varepsilon) \boldsymbol{\gamma}^{\varepsilon}(\mathbf{v}) \mathbf{Q}(\varepsilon)^{T}\right) \sqrt{g(\varepsilon)} d z \\
& \quad-\alpha \int_{\Omega} p(\varepsilon) \operatorname{tr}\left(\mathbf{Q}(\varepsilon) \boldsymbol{\gamma}^{\varepsilon}(\mathbf{v}) \mathbf{Q}(\varepsilon)^{T}\right) \sqrt{g(\varepsilon)} d z=\int_{\Sigma_{ \pm}} \mathcal{P}_{ \pm} \cdot \mathbf{v} \sqrt{g(\varepsilon)} d s, \\
& \quad \mathbf{v} \in H_{0}^{1}\left(\omega ; \mathbb{R}^{3}\right), \text { a.e. } t \in[0, T], \\
& \int_{\Omega} \beta \frac{\partial p(\varepsilon)}{\partial t} q \sqrt{g(\varepsilon)} d z+\int_{\Omega} \alpha \frac{\partial}{\partial t} \operatorname{tr}\left(\mathbf{Q}(\varepsilon) \boldsymbol{\gamma}^{\varepsilon}(\mathbf{u}(\varepsilon)) \mathbf{Q}(\varepsilon)^{T}\right) q \sqrt{g(\varepsilon)} d z \\
& +\varepsilon^{2} \int_{\Omega} \mathbf{Q}(\varepsilon) \nabla^{\varepsilon} p(\varepsilon) \cdot \mathbf{Q}(\varepsilon) \nabla^{\varepsilon} q \sqrt{g(\varepsilon)} d z=\mp \int_{\Sigma_{ \pm}} V q \sqrt{g(\varepsilon)} d s, \quad q \in H^{1}(\Omega) .
\end{aligned}
$$

In the limit when $\varepsilon \rightarrow 0$ we obtain

$$
\begin{aligned}
& \int_{\Omega} \mathcal{C}\left(\mathbf{Q} \gamma^{0} \mathbf{Q}^{T}\right):\left(\mathbf{Q}\left(\gamma_{y}(\mathbf{v})-\sum_{i=1}^{3} v_{i} \boldsymbol{\Gamma}^{i}(0)\right) \mathbf{Q}^{T}\right) \sqrt{a} d z \\
& -\alpha \int_{\Omega} p^{0} \operatorname{tr}\left(\mathbf{Q}\left(\gamma_{y}(\mathbf{v})-\sum_{i=1}^{3} v_{i} \boldsymbol{\Gamma}^{i}(0)\right) \mathbf{Q}^{T}\right) \sqrt{a} d z=\int_{\Sigma_{ \pm}} \mathcal{P}_{ \pm} \cdot \mathbf{v} \sqrt{a} d s \\
& \quad \mathbf{v} \in H_{0}^{1}\left(\omega ; \mathbb{R}^{3}\right), \text { a.e. } t \in[0, T], \\
& \int_{\Omega} \beta \frac{\partial p^{0}}{\partial t} q \sqrt{a} d z+\int_{\Omega} \alpha \frac{\partial}{\partial t} \operatorname{tr}\left(\mathbf{Q} \gamma^{0} \mathbf{Q}^{T}\right) q \sqrt{a} d z+\int_{\Omega} \frac{\partial p^{0}}{\partial z_{3}} \mathbf{Q e}_{3} \cdot \frac{\partial q}{\partial z_{3}} \mathbf{Q e}_{3} \sqrt{a} d z \\
& =\mp \int_{\Sigma_{ \pm}} V q \sqrt{a} d s, \quad q \in H^{1}(\Omega) .
\end{aligned}
$$

Note that $\mathbf{Q e}_{3} \cdot \mathbf{Q} \mathbf{e}_{3}=1$. Since

$$
\gamma_{y}(\mathbf{v})-v_{i} \boldsymbol{\Gamma}^{i}(0)=\left[\begin{array}{cc}
\gamma(\mathbf{v}) & \frac{1}{2} \partial_{1} v_{3}+\sum_{\sigma=1}^{2} v_{\sigma} b_{1}^{\sigma} \\
\frac{1}{2} \partial_{1} v_{3}+\sum_{\sigma=1}^{2} v_{\sigma} b_{1}^{\sigma} & \frac{1}{2} \partial_{2} v_{3}+\sum_{\sigma=1}^{2} v_{\sigma} b_{2}^{\sigma}+\sum_{\sigma=1}^{2} v_{\sigma} b_{2}^{\sigma} \\
0 & \frac{1}{2} \partial_{2}
\end{array}\right]
$$

using (5.1) we get

$$
\operatorname{tr}\left(\mathbf{Q}\left(\boldsymbol{\gamma}_{y}(\mathbf{v})-\sum_{i=1}^{3} v_{i} \boldsymbol{\Gamma}^{i}(0)\right) \mathbf{Q}^{T}\right)=\operatorname{tr}\left(\mathbf{Q}^{T} \mathbf{Q}\left(\boldsymbol{\gamma}_{y}(\mathbf{v})-\sum_{i=1}^{3} v_{i} \boldsymbol{\Gamma}^{i}(0)\right)\right)=\mathbf{A}^{c}: \boldsymbol{\gamma}(\mathbf{v}) .
$$

Next, using Lemma 19 yields

$$
\begin{aligned}
\operatorname{tr}\left(\mathbf{Q} \gamma^{0} \mathbf{Q}^{T}\right) & =\operatorname{tr}\left(\mathbf{Q}^{T} \mathbf{Q} \boldsymbol{\gamma}^{0}\right)=\mathbf{A}^{c}: \boldsymbol{\gamma}(\mathbf{u})+\gamma_{33}^{0} \\
& =\mathbf{A}^{c}: \boldsymbol{\gamma}(\mathbf{u})+\frac{\alpha}{\tilde{\lambda}+2} p^{0}-\frac{\tilde{\lambda}}{\tilde{\lambda}+2} \mathbf{A}^{c}: \boldsymbol{\gamma}(\mathbf{u})=\frac{2}{\tilde{\lambda}+2} \mathbf{A}^{c}: \boldsymbol{\gamma}(\mathbf{u})+\frac{\alpha}{\tilde{\lambda}+2} p^{0} .
\end{aligned}
$$

Further, using (5.1) and Lemma 19 we compute

$$
\mathbf{Q}^{T} \mathbf{Q} \boldsymbol{\gamma}^{0} \mathbf{Q}^{T} \mathbf{Q}=\left[\begin{array}{cc}
\mathbf{A}^{c} \boldsymbol{\gamma}(\mathbf{u}) \mathbf{A}^{c} & 0 \\
0 & \gamma_{33}^{0}
\end{array}\right]=\left[\begin{array}{cc}
\mathbf{A}^{c} \boldsymbol{\gamma}(\mathbf{u}) \mathbf{A}^{c} & 0 \\
0 & \frac{\alpha}{\tilde{\lambda}+2} p^{0}-\frac{\tilde{\lambda}}{\tilde{\lambda}+2} \mathbf{A}^{c}: \boldsymbol{\gamma}(\mathbf{u})
\end{array}\right] .
$$


Now the main elastic term in the first equation in (5.4) is computed:

$$
\begin{aligned}
\int_{\Omega} \mathcal{C} & \left(\mathbf{Q} \gamma^{0} \mathbf{Q}^{T}\right):\left(\mathbf{Q}\left(\gamma_{y}(\mathbf{v})-\sum_{i=1}^{3} v_{i} \boldsymbol{\Gamma}^{i}(0)\right) \mathbf{Q}^{T}\right) \sqrt{a} d z \\
= & \int_{\Omega} \tilde{\lambda} \operatorname{tr}\left(\mathbf{Q} \gamma^{0} \mathbf{Q}^{T}\right) \operatorname{tr}\left(\mathbf{Q}\left(\boldsymbol{\gamma}_{y}(\mathbf{v})-\sum_{i=1}^{3} v_{i} \boldsymbol{\Gamma}^{i}(0)\right) \mathbf{Q}^{T}\right)+2 \mathbf{Q} \boldsymbol{\gamma}^{0} \mathbf{Q}^{T}: \mathbf{Q}\left(\boldsymbol{\gamma}_{y}(\mathbf{v})-\sum_{i=1}^{3} v_{i} \boldsymbol{\Gamma}^{i}(0)\right) \mathbf{Q}^{T} \sqrt{a} d z \\
= & \int_{\Omega} \tilde{\lambda}\left(\frac{2}{\tilde{\lambda}+2} \mathbf{A}^{c}: \boldsymbol{\gamma}(\mathbf{u})+\frac{\alpha}{\tilde{\lambda}+2} p^{0}\right) \mathbf{A}^{c}: \boldsymbol{\gamma}(\mathbf{v}) \sqrt{a} d z \\
& +\int_{\Omega} 2 \mathbf{Q}^{T} \mathbf{Q} \boldsymbol{\gamma}^{0} \mathbf{Q}^{T} \mathbf{Q}:\left(\boldsymbol{\gamma}_{y}(\mathbf{v})-\sum_{i=1}^{3} v_{i} \boldsymbol{\Gamma}^{i}(0)\right) \sqrt{a} d z \\
= & \int_{\Omega} \frac{2 \tilde{\lambda}}{\tilde{\lambda}+2}\left(\mathbf{A}^{c}: \boldsymbol{\gamma}(\mathbf{u})\right)\left(\mathbf{A}^{c}: \boldsymbol{\gamma}(\mathbf{v})\right)+\frac{\tilde{\lambda} \alpha}{\tilde{\lambda}+2} p^{0} \mathbf{A}^{c}: \boldsymbol{\gamma}(\mathbf{v}) \sqrt{a} d z+\int_{\Omega} 2 \mathbf{A}^{c} \boldsymbol{\gamma}(\mathbf{u}) \mathbf{A}^{c}: \boldsymbol{\gamma}(\mathbf{v}) \sqrt{a} d z
\end{aligned}
$$

Let us define the tensor (it usually appears in plate and shell theories!)

$$
\tilde{\mathcal{C}} \mathbf{E}=\frac{2 \tilde{\lambda}}{\tilde{\lambda}+2} \operatorname{tr}(\mathbf{E}) \mathbf{I}+2 \mathbf{E}, \quad \mathbf{E} \in \mathbb{R}_{\text {sym }}^{2 \times 2}
$$

The first equation in (5.4) now becomes: for all $\mathbf{v} \in H_{0}^{1}\left(\omega ; \mathbb{R}^{3}\right)$ one has

$$
\begin{aligned}
\int_{\omega} \tilde{\mathcal{C}}\left(\mathbf{A}^{c} \boldsymbol{\gamma}(\mathbf{u})\right): \gamma(\mathbf{v}) \mathbf{A}^{c} \sqrt{a} d z_{1} d z_{2}+\int_{\Omega} \frac{\tilde{\lambda} \alpha}{\tilde{\lambda}+2} p^{0} \mathbf{A}^{c}: \gamma(\mathbf{v}) \sqrt{a} d z-\alpha \int_{\Omega} p^{0} \mathbf{A}^{c}: \gamma(\mathbf{v}) \sqrt{a} d z \\
=\int_{\omega}\left(\mathcal{P}_{+}+\mathcal{P}_{-}\right) \cdot \mathbf{v} \sqrt{a} d z_{1} d z_{2} .
\end{aligned}
$$

By density of $H_{0}^{1}\left(\omega ; \mathbb{R}^{3}\right)$ in $\mathcal{V}_{M}(\omega)$ the above equation implies:

$$
\begin{gathered}
\int_{\omega} \tilde{\mathcal{C}}\left(\mathbf{A}^{c} \boldsymbol{\gamma}(\mathbf{u})\right): \gamma(\mathbf{v}) \mathbf{A}^{c} \sqrt{a} d z_{1} d z_{2}-\frac{2 \alpha}{\tilde{\lambda}+2} \int_{\omega} \int_{-1 / 2}^{1 / 2} p^{0} d z_{3} \mathbf{A}^{c}: \gamma(\mathbf{v}) \sqrt{a} d z_{1} d z_{2} \\
=\int_{\omega}\left(\mathcal{P}_{+}+\mathcal{P}_{-}\right) \cdot \mathbf{v} \sqrt{a} d z_{1} d z_{2} ., \quad \mathbf{v} \in \mathcal{V}_{M}(\omega) .
\end{gathered}
$$

Equation (5.8) is the classical equation of the membrane shell model with the addition of the pressure $p^{0}$ term.

Using (5.6), the second equation in (5.4) can be now written by

$$
\begin{aligned}
\int_{\Omega} & \left(\beta+\frac{\alpha^{2}}{\tilde{\lambda}+2}\right) \frac{\partial p^{0}}{\partial t} q \sqrt{a} d z+\int_{\Omega} \alpha \frac{\partial}{\partial t}\left(\frac{2}{\tilde{\lambda}+2} \mathbf{A}^{c}: \gamma(\mathbf{u})\right) q \sqrt{a} d z+\int_{\Omega} \frac{\partial p^{0}}{\partial z_{3}} \frac{\partial q}{\partial z_{3}} \sqrt{a} d z \\
= & \mp \int_{\Sigma_{ \pm}} V q \sqrt{a} d s, \quad q \in H^{1}(\Omega) .
\end{aligned}
$$

The elliptic membrane poroelastic shell model is given by (5.8), (5.9).

STEP 3 (The strong convergences of strain and pressure).

As mentioned in Remark 8 the limit problem can be decoupled and the displacement can be calculated independently of the pressure, by solving the elliptic membrane shell model with modified coefficients. However the same decoupling cannot be done for the three-dimensional problem and thus the result of the strong convergence for the classical elliptic membrane shell derivation, see [5], 
cannot be applied directly to the poroelastic case. Hence we adapt the ideas from [5]. We start with

$$
\begin{aligned}
\Lambda(\varepsilon)(t)= & \frac{1}{2} \int_{\Omega} \mathcal{C}\left(\mathbf{Q}(\varepsilon)\left(\gamma^{\varepsilon}(\mathbf{u}(\varepsilon))(t)-\boldsymbol{\gamma}^{0}(t)\right) \mathbf{Q}(\varepsilon)^{T}\right) \\
& :\left(\mathbf{Q}(\varepsilon)\left(\boldsymbol{\gamma}^{\varepsilon}(\mathbf{u}(\varepsilon))(t)-\gamma^{0}(t)\right) \mathbf{Q}(\varepsilon)^{T}\right) \sqrt{g(\varepsilon)}+\frac{1}{2} \beta \int_{\Omega}\left(p(\varepsilon)(t)-p^{0}(t)\right)^{2} \sqrt{g(\varepsilon)} d z \\
& +\varepsilon^{2} \int_{0}^{t} \int_{\Omega}\left(\nabla^{\varepsilon} p(\varepsilon)-\nabla^{\varepsilon} p^{0}\right) \mathbf{Q}(\varepsilon)^{T} \cdot\left(\nabla^{\varepsilon} p(\varepsilon)-\nabla^{\varepsilon} p^{0}\right) \mathbf{Q}(\varepsilon)^{T} \sqrt{g(\varepsilon)} d z .
\end{aligned}
$$

and we will show that $\Lambda(\varepsilon)(t) \rightarrow \Lambda(t)$ as $\varepsilon$ tends to zero for all $t \in[0, T]$. Since $\Lambda(\varepsilon) \geq 0$ the $\Lambda \geq 0$ as well. After some calculation we will show that actually $\Lambda=0$. This will give the strong convergences in (4.7).

Since we have only weak convergences in (4.7) we first remove quadratic terms in $\Lambda(\varepsilon)$ using (3.16) for $\mathbf{v}=\frac{\partial \mathbf{u}(\varepsilon)}{\partial t}$ and $q=p(\varepsilon)$ divided by $\varepsilon$. Integration of (3.16) over time, using $\left.p(\varepsilon)\right|_{t=0}=0$ and $\left.\mathbf{u}(\varepsilon)\right|_{t=0}=0$, implies

$$
\begin{aligned}
& \frac{1}{2} \int_{\Omega} \mathcal{C}\left(\mathbf{Q}(\varepsilon) \boldsymbol{\gamma}^{\varepsilon}(\mathbf{u}(\varepsilon)) \mathbf{Q}(\varepsilon)^{T}\right):\left(\mathbf{Q}(\varepsilon) \boldsymbol{\gamma}^{\varepsilon}(\mathbf{u}(\varepsilon)) \mathbf{Q}(\varepsilon)^{T}\right) \sqrt{g(\varepsilon)} d z \\
& \quad+\frac{1}{2} \beta \int_{\Omega} p(\varepsilon)^{2} \sqrt{g(\varepsilon)} d z+\varepsilon^{2} \int_{0}^{t} \int_{\Omega} \mathbf{Q}(\varepsilon) \nabla^{\varepsilon} p(\varepsilon) \cdot \mathbf{Q}(\varepsilon) \nabla^{\varepsilon} p(\varepsilon) \sqrt{g(\varepsilon)} d z d \tau \\
& =\int_{0}^{t} \int_{\Sigma_{ \pm}} \mathcal{P}_{ \pm} \cdot \frac{\partial \mathbf{u}(\varepsilon)}{\partial t} \sqrt{g(\varepsilon)} d s d \tau \mp \int_{0}^{t} \int_{\Sigma_{ \pm}} V p(\varepsilon) \sqrt{g(\varepsilon)} d s d \tau
\end{aligned}
$$

Inserting this into the definition of $\Lambda(\varepsilon)$ we obtain

$$
\begin{aligned}
\Lambda(\varepsilon)(t)= & \int_{0}^{t} \int_{\Sigma_{ \pm}} \mathcal{P}_{ \pm} \cdot \frac{\partial \mathbf{u}(\varepsilon)}{\partial t} \sqrt{g(\varepsilon)} d s d \tau \mp \int_{0}^{t} \int_{\Sigma_{ \pm}} V p(\varepsilon) \sqrt{g(\varepsilon)} d s d \tau \\
& -\int_{\Omega} \mathcal{C}\left(\mathbf{Q}(\varepsilon) \boldsymbol{\gamma}^{\varepsilon}(\mathbf{u}(\varepsilon))(t) \mathbf{Q}(\varepsilon)^{T}\right):\left(\mathbf{Q}(\varepsilon) \boldsymbol{\gamma}^{0}(t) \mathbf{Q}(\varepsilon)^{T}\right) \sqrt{g(\varepsilon)} d z \\
& -\beta \int_{\Omega} p(\varepsilon) p^{0} \sqrt{g(\varepsilon)} d z \\
& -2 \varepsilon^{2} \int_{0}^{t} \int_{\Omega} \mathbf{Q}(\varepsilon) \nabla^{\varepsilon} p(\varepsilon)(t) \cdot \mathbf{Q}(\varepsilon) \nabla^{\varepsilon} p^{0}(t) \sqrt{g(\varepsilon)} d z d \tau \\
& +\frac{1}{2} \int_{\Omega} \mathcal{C}\left(\mathbf{Q}(\varepsilon) \boldsymbol{\gamma}^{0} \mathbf{Q}(\varepsilon)^{T}\right):\left(\mathbf{Q}(\varepsilon) \boldsymbol{\gamma}^{0} \mathbf{Q}(\varepsilon)^{T}\right) \sqrt{g(\varepsilon)} d z+\frac{1}{2} \beta \int_{\Omega}\left(p^{0}\right)^{2} \sqrt{g(\varepsilon)} d z \\
& +\varepsilon^{2} \int_{0}^{t} \int_{\Omega} \mathbf{Q}(\varepsilon) \nabla^{\varepsilon} p^{0} \cdot \mathbf{Q}(\varepsilon) \nabla^{\varepsilon} p^{0} \sqrt{g(\varepsilon)} d z d \tau
\end{aligned}
$$

Now we take the limit as $\varepsilon$ tends to zero and obtain that $\Lambda(\varepsilon)(t) \rightarrow \Lambda(t) \geq 0$, where

$$
\begin{aligned}
& \Lambda(t)=\int_{0}^{t} \int_{\omega}\left(\mathcal{P}_{+}+\mathcal{P}_{-}\right) \cdot \frac{\partial \mathbf{u}}{\partial t} \sqrt{a} d s d \tau \mp \int_{0}^{t} \int_{\Sigma_{ \pm}} V p^{0} \sqrt{a} d s d \tau \\
& -\frac{1}{2} \int_{\Omega} \mathcal{C}\left(\mathbf{Q} \gamma^{0}(t) \mathbf{Q}^{T}\right):\left(\mathbf{Q} \gamma^{0}(t) \mathbf{Q}^{T}\right) \sqrt{a} d z-\frac{1}{2} \beta \int_{\Omega} p^{0}(t)^{2} \sqrt{a} d z-\int_{0}^{t} \int_{\Omega}\left(\frac{\partial p^{0}}{\partial z_{3}}\right)^{2} \sqrt{a} d z d \tau .
\end{aligned}
$$

We now insert $\frac{\partial \mathbf{u}}{\partial t}$ as a test function in (5.8), $p^{0}$ in (5.9) and sum up the equations. The antisymmetric terms cancel out as before. Then we integrate the equation over time and use the initial 
conditions to obtain

$$
\begin{aligned}
& \frac{1}{2} \int_{\omega} \tilde{\mathcal{C}}\left(\mathbf{A}^{c} \gamma(\mathbf{u}(t))\right):\left(\gamma(\mathbf{u}(t)) \mathbf{A}^{c}\right) \sqrt{a} d z_{1} d z_{2}+\frac{1}{2} \int_{\Omega}\left(\beta+\frac{\alpha^{2}}{\tilde{\lambda}+2}\right)\left(p^{0}(t)\right)^{2} \sqrt{a} d z \\
& \quad+\int_{0}^{t} \int_{\Omega}\left(\frac{\partial p^{0}}{\partial z_{3}}\right)^{2} \sqrt{a} d z d \tau=\int_{0}^{t} \int_{\omega}\left(\mathcal{P}_{+}+\mathcal{P}_{-}\right) \cdot \frac{\partial \mathbf{u}}{\partial t} \sqrt{a} d s d \tau \mp \int_{0}^{t} \int_{\Sigma_{ \pm}} V p^{0} \sqrt{a} d s d \tau .
\end{aligned}
$$

Inserting the above equation into (5.10) yields

$$
\begin{aligned}
\Lambda(t)= & \frac{1}{2} \int_{\omega} \tilde{\mathcal{C}}\left(\mathbf{A}^{c} \boldsymbol{\gamma}(\mathbf{u}(t))\right):\left(\boldsymbol{\gamma}(\mathbf{u}(t)) \mathbf{A}^{c}\right) \sqrt{a} d z_{1} d z_{2}+\frac{1}{2} \int_{\Omega} \frac{\alpha^{2}}{\tilde{\lambda}+2}\left(p^{0}(t)\right)^{2} \sqrt{a} d z \\
& -\frac{1}{2} \int_{\Omega} \mathcal{C}\left(\mathbf{Q} \gamma^{0}(t) \mathbf{Q}^{T}\right):\left(\mathbf{Q} \boldsymbol{\gamma}^{0}(t) \mathbf{Q}^{T}\right) \sqrt{a} d z .
\end{aligned}
$$

Next we compute the elastic energy using (5.3), (5.6), (5.7):

$$
\begin{aligned}
& \int_{\Omega} \mathcal{C}\left(\mathbf{Q} \gamma^{0} \mathbf{Q}^{T}\right):\left(\mathbf{Q} \boldsymbol{\gamma}^{0} \mathbf{Q}^{T}\right) \sqrt{a} d z \\
& =\int_{\Omega} \tilde{\lambda}\left(\operatorname{tr}\left(\mathbf{Q} \gamma^{0} \mathbf{Q}^{T}\right)\right)^{2}+2 \mathbf{Q}^{T} \mathbf{Q} \boldsymbol{\gamma}^{0} \mathbf{Q}^{T} \mathbf{Q}: \boldsymbol{\gamma}^{0} \sqrt{a} d z \\
& =\int_{\Omega} \tilde{\lambda}\left(\frac{2}{\tilde{\lambda}+2} \mathbf{A}^{c}: \boldsymbol{\gamma}(\mathbf{u})+\frac{\alpha}{\tilde{\lambda}+2} p^{0}\right)^{2}+2\left[\begin{array}{cc}
\mathbf{A}^{c} \boldsymbol{\gamma}(\mathbf{u}) \mathbf{A}^{c} & 0 \\
0 & \frac{\alpha}{\tilde{\lambda}+2} p^{0}-\frac{\tilde{\lambda}}{\tilde{\lambda}+2} \mathbf{A}^{c}: \boldsymbol{\gamma}(\mathbf{u})
\end{array}\right]: \boldsymbol{\gamma}^{0} \sqrt{a} d z \\
& =\int_{\Omega} \tilde{\lambda}\left(\frac{2}{\tilde{\lambda}+2} \mathbf{A}^{c}: \gamma(\mathbf{u})+\frac{\alpha}{\tilde{\lambda}+2} p^{0}\right)^{2} \\
& +2\left(\mathbf{A}^{c} \boldsymbol{\gamma}(\mathbf{u}) \mathbf{A}^{c}: \boldsymbol{\gamma}(\mathbf{u})+\left(\frac{\alpha}{\tilde{\lambda}+2} p^{0}-\frac{\tilde{\lambda}}{\tilde{\lambda}+2} \mathbf{A}^{c}: \boldsymbol{\gamma}(\mathbf{u})\right)^{2}\right) \sqrt{a} d z \\
& =\int_{\Omega}\left(\frac{4 \tilde{\lambda}}{(\tilde{\lambda}+2)^{2}}\left(\mathbf{A}^{c}: \boldsymbol{\gamma}(\mathbf{u})\right)^{2}+\frac{4 \tilde{\lambda} \alpha}{(\tilde{\lambda}+2)^{2}} \mathbf{A}^{c}: \boldsymbol{\gamma}(\mathbf{u}) p^{0}+\frac{\tilde{\lambda} \alpha^{2}}{(\tilde{\lambda}+2)^{2}}\left(p^{0}\right)^{2}+2 \mathbf{A}^{c} \boldsymbol{\gamma}(\mathbf{u}) \mathbf{A}^{c}: \boldsymbol{\gamma}(\mathbf{u})\right. \\
& \left.+\frac{2 \alpha^{2}}{(\tilde{\lambda}+2)^{2}}\left(p^{0}\right)^{2}-\frac{4 \tilde{\lambda} \alpha}{(\tilde{\lambda}+2)^{2}} \mathbf{A}^{c}: \gamma(\mathbf{u}) p^{0}+\frac{2 \tilde{\lambda}^{2}}{(\tilde{\lambda}+2)^{2}}\left(\mathbf{A}^{c}: \gamma(\mathbf{u})\right)^{2}\right) \sqrt{a} d z \\
& =\int_{\Omega} \frac{2 \tilde{\lambda}}{\tilde{\lambda}+2}\left(\mathbf{A}^{c}: \boldsymbol{\gamma}(\mathbf{u})\right)^{2}+\frac{\alpha^{2}}{\tilde{\lambda}+2}\left(p^{0}\right)^{2}+2 \mathbf{A}^{c} \boldsymbol{\gamma}(\mathbf{u}) \mathbf{A}^{c}: \boldsymbol{\gamma}(\mathbf{u}) \sqrt{a} d z \\
& =\int_{\Omega} \tilde{\mathcal{C}}\left(\mathbf{A}^{c} \boldsymbol{\gamma}(\mathbf{u})\right): \boldsymbol{\gamma}(\mathbf{u}) \mathbf{A}^{c}+\frac{\alpha^{2}}{\tilde{\lambda}+2}\left(p^{0}\right)^{2} \sqrt{a} d z .
\end{aligned}
$$

Inserting this into (5.11) we obtain that $\Lambda(t)=0$. We now have $\Lambda(\varepsilon)(t) \rightarrow 0$ for every $t \in[0, T]$. Since $\Lambda(\varepsilon):[0, T] \rightarrow \mathbb{R}$ is an equicontinuous family, we conclude strong convergences of the strain tensor and the pressure

$$
\begin{aligned}
& \gamma^{\varepsilon}(\mathbf{u}(\varepsilon)) \rightarrow \gamma^{0} \quad \text { strongly in } C\left([0, T] ; L^{2}\left(\Omega ; \mathbb{R}^{3 \times 3}\right)\right), \\
& p(\varepsilon) \rightarrow p^{0} \quad \text { strongly in } C\left([0, T] ; L^{2}(\Omega)\right), \\
& \frac{\partial p(\varepsilon)}{\partial z_{3}} \rightarrow \frac{\partial p^{0}}{\partial z_{3}} \quad \text { strongly in } L^{2}\left(0, T ; L^{2}(\Omega)\right) .
\end{aligned}
$$

STEP 4 (The strong convergences of the displacements). The setting is more complicated than in the proof of Theorem 4.4-1 from [5] because the problem is time dependent. The first convergence in (5.12) implies

$$
\gamma_{\alpha \beta}(\mathbf{u}(\varepsilon)) \rightarrow \gamma_{\alpha \beta}(\mathbf{u}) \text { strongly in } C\left([0, T] ; L^{2}(\Omega)\right), \quad \alpha, \beta \in\{1,2\} .
$$


Let us now denote by ${ }^{-}$the operator of averaging over $z_{3}$, i.e.,

$$
\bar{v}(\cdot)=\int_{-1 / 2}^{1 / 2} v\left(\cdot, z_{3}\right) d z_{3} .
$$

Then from (5.13) we obtain

$$
\gamma_{\alpha \beta}(\overline{\mathbf{u}(\varepsilon)}) \rightarrow \gamma_{\alpha \beta}(\overline{\mathbf{u}}) \text { strongly in } C\left([0, T] ; L^{2}(\omega)\right), \quad \alpha, \beta \in\{1,2\} .
$$

Note that $\overline{\mathbf{u}}=\mathbf{u}$ since $\mathbf{u}$ is independent of $z_{3}$. The inequality of Korn's type on an elliptic surface, see Lemma 6 ([5, Theorem 2.7-3]), for $\overline{\mathbf{u}(\varepsilon)}-\overline{\mathbf{u}}$ implies

$$
\left\|\overline{u_{1}(\varepsilon)}-u_{1}\right\|_{H^{1}(\omega)}^{2}+\left\|\overline{u_{2}(\varepsilon)}-u_{2}\right\|_{H^{1}(\omega)}^{2}+\left\|\overline{u_{3}(\varepsilon)}-u_{3}\right\|_{L^{2}(\omega)}^{2} \leq C_{M}\|\gamma(\overline{\mathbf{u}(\varepsilon)})-\gamma(\overline{\mathbf{u}})\|_{L^{2}\left(\Omega ; \mathbb{R}^{3 \times 3}\right)}^{2} .
$$

Application of (5.13) yields

$$
\begin{aligned}
& \overline{u_{\alpha}(\varepsilon)} \rightarrow u_{\alpha} \text { strongly in } C\left([0, T] ; H^{1}(\omega)\right), \quad \alpha \in\{1,2\}, \\
& \overline{u_{3}(\varepsilon)} \rightarrow u_{3} \text { strongly in } C\left([0, T] ; L^{2}(\omega)\right) .
\end{aligned}
$$

Next we prove that

$$
u_{3}(\varepsilon) \rightarrow u_{3} \text { strongly in } C\left([0, T] ; L^{2}(\Omega)\right) .
$$

From the Poincare type estimate

$$
\left|u_{3}(\varepsilon)\left(\cdot, z_{3}\right)-\overline{u_{3}(\varepsilon)}(\cdot)\right| \leq C \sqrt{\int_{-1 / 2}^{1 / 2}\left(\partial_{3} u_{3}(\varepsilon)\left(\cdot, y_{3}\right)\right)^{2} d y_{3}}
$$

we obtain

$$
\left\|u_{3}(\varepsilon)-\overline{u_{3}(\varepsilon)}\right\|_{C\left([0, T] ; L^{2}(\Omega)\right)} \leq C\left\|\partial_{3} u_{3}(\varepsilon)\right\|_{C\left([0, T] ; L^{2}(\Omega)\right)} .
$$

From $\gamma_{33}^{\varepsilon}(\mathbf{u}(\varepsilon))=\frac{1}{\varepsilon} \partial_{3} u_{3}(\varepsilon) \rightarrow \gamma_{33}^{0}$ strongly in $C\left([0, T] ; L^{2}(\Omega)\right)$ we conclude $\partial_{3} u_{3}(\varepsilon) \rightarrow 0$ strongly in $C\left([0, T] ; L^{2}(\Omega)\right)$. Together with the last convergence in (5.14) this implies (5.15).

In the remaining part of the proof we prove

$$
u_{\alpha}(\varepsilon) \rightarrow u_{\alpha} \text { strongly in } C\left([0, T] ; H^{1}(\Omega)\right), \quad \alpha \in\{1,2\} .
$$

It follows from the Korn inequality and the Lions lemma (see [14]). Let us denote $\mathbf{u}^{\prime}(\varepsilon)=\left(u_{1}(\varepsilon), u_{2}(\varepsilon), 0\right)$, $\mathbf{u}^{\prime}=\left(u_{1}, u_{2}, 0\right)$. Then, using the Korn inequality, the convergence

$$
\mathbf{e}\left(\mathbf{u}^{\prime}(\varepsilon)\right) \rightarrow \mathbf{e}\left(\mathbf{u}^{\prime}\right) \text { strongly in } C\left([0, T] ; L^{2}\left(\Omega ; \mathbb{R}^{3 \times 3}\right)\right)
$$

is equivalent to (5.16) (e(u) denotes the symmetrized gradient). Convergence in (5.17) will be obtained component by component. Since for $\alpha, \beta \in\{1,2\}$

$$
e_{\alpha \beta}\left(\mathbf{u}^{\prime}(\varepsilon)\right)=\gamma_{\alpha \beta}^{\varepsilon}(\mathbf{u}(\varepsilon))+\sum_{i=1}^{3} u_{i}(\varepsilon) \Gamma_{\alpha \beta}^{i}(\varepsilon),
$$

using the first convergence in (5.12) for $\gamma_{\alpha \beta}^{\varepsilon}(\mathbf{u}(\varepsilon))$, Remark 18 for $u_{1}(\varepsilon)$ and $u_{2}(\varepsilon)$, (5.15) for $u_{3}(\varepsilon)$ and (4.3) and (4.4) we obtain

$$
e_{\alpha \beta}\left(\mathbf{u}^{\prime}(\varepsilon)\right) \rightarrow \gamma_{\alpha \beta}(\mathbf{u})+\sum_{i=1}^{3} u_{i} \Gamma_{\alpha \beta}^{i}(0)=e_{\alpha \beta}\left(\mathbf{u}^{\prime}\right) \text { strongly in } C\left([0, T] ; L^{2}(\Omega)\right), \quad \alpha, \beta=1,2 .
$$

Since $e_{33}\left(\mathbf{u}^{\prime}(\varepsilon)\right)=e_{33}\left(\mathbf{u}^{\prime}\right)=0$ the convergence of this component is trivial. 
that

For the convergence of $e_{\alpha 3}\left(\mathbf{u}^{\prime}(\varepsilon)\right)=\frac{1}{2} \partial_{3} u_{\alpha}(\varepsilon)$ we apply the Lions lemma. Thus we first prove

$$
\partial_{3} u_{\alpha}(\varepsilon), \partial_{13} u_{\alpha}(\varepsilon), \partial_{23} u_{\alpha}(\varepsilon), \partial_{33} u_{\alpha}(\varepsilon) \rightarrow 0 \text { strongly in } C\left([0, T] ; H^{-1}(\Omega)\right), \quad \alpha=1,2 .
$$

We start with the expression

$$
\frac{1}{\varepsilon} \partial_{3} u_{\alpha}(\varepsilon)=2 \gamma_{\alpha 3}^{\varepsilon}(\mathbf{u}(\varepsilon))-\partial_{\alpha} u_{3}(\varepsilon)+2 \sum_{i=1}^{3} u_{i}(\varepsilon) \Gamma_{\alpha 3}^{i}(\varepsilon) .
$$

The first and the third term on the right hand side converge strongly in $C\left([0, T] ; L^{2}(\Omega)\right)$, while the middle one converges only in $C\left([0, T] ; H^{-1}(\Omega)\right)$ by (5.15). Thus we obtain that $\partial_{3} u_{\alpha}(\varepsilon) \rightarrow 0$ in $C\left([0, T] ; H^{-1}(\Omega)\right)$. Differentiating $(5.20)$ with respect to $z_{3}$ yields

$$
\frac{1}{\varepsilon} \partial_{33} u_{\alpha}(\varepsilon)=2 \partial_{3} \gamma_{\alpha 3}^{\varepsilon}(\mathbf{u}(\varepsilon))-\partial_{\alpha} \partial_{3} u_{3}(\varepsilon)+2 \sum_{i=1}^{3} \partial_{3}\left(u_{i}(\varepsilon) \Gamma_{\alpha 3}^{i}(\varepsilon)\right) .
$$

Since $\partial_{3} u_{3}(\varepsilon) \rightarrow 0$ in $C\left([0, T] ; L^{2}(\Omega)\right)$ the convergence of $\partial_{33} u_{\alpha}(\varepsilon)$ in $C\left([0, T] ; H^{-1}(\Omega)\right)$ is obtained. Differentiating (5.18) with respect to $z_{3}$ for $\alpha=\beta$ yields

$$
\partial_{\alpha 3} u_{\alpha}(\varepsilon)=\partial_{3} e_{\alpha \alpha}\left(\mathbf{u}^{\prime}(\varepsilon)\right) \rightarrow \partial_{3} e_{\alpha \alpha}\left(\mathbf{u}^{\prime}\right)=0 \text { strongly in } C\left([0, T] ; H^{-1}(\Omega)\right), \quad \alpha=1,2 .
$$

Since

$$
\partial_{13} u_{2}(\varepsilon)=\varepsilon \partial_{1} \gamma_{23}^{\varepsilon}(\mathbf{u}(\varepsilon))-\varepsilon \partial_{2} \gamma_{13}^{\varepsilon}(\mathbf{u}(\varepsilon))+\partial_{3} e_{12}\left(\mathbf{u}^{\prime}(\varepsilon)\right)+\varepsilon\left(\partial_{1} \sum_{\tau=1}^{2} u_{\tau}(\varepsilon) \Gamma_{23}^{\tau}(\varepsilon)-\partial_{2} \sum_{\tau=1}^{2} u_{\tau}(\varepsilon) \Gamma_{13}^{\tau}(\varepsilon)\right)
$$

and since all terms on the right hand side strongly converge in $C\left([0, T] ; H^{-1}(\Omega)\right)$ we obtain the strong convergence of $\partial_{13} u_{2}(\varepsilon)$ in the same space. It then implies that the term

$$
\partial_{23} u_{1}(\varepsilon)=2 \partial_{3} \gamma_{12}^{\varepsilon}(\mathbf{u}(\varepsilon))-\partial_{13} u_{2}(\varepsilon)+2 \partial_{3} \sum_{i=1}^{3} u_{i}(\varepsilon) \Gamma_{12}^{i}(\varepsilon)
$$

converges strongly in $C\left([0, T] ; H^{-1}(\Omega)\right)$. Thus we have proved (5.19). A consequence of Lions lemma is that the spaces

$$
v \in L^{2}(\Omega) \leftrightarrow\left(v, \partial_{1} v, \partial_{2} v, \partial_{3} v\right) \in H^{-1}(\Omega)^{4}
$$

are isomorphic. Therefore the spaces

$$
v \in C\left([0, T] ; L^{2}(\Omega)\right) \leftrightarrow\left(v, \partial_{1} v, \partial_{2} v, \partial_{3} v\right) \in C\left([0, T] ; H^{-1}(\Omega)\right)^{4}
$$

are also isomorphic. Therefore (5.19) implies that $e_{\alpha 3}\left(\mathbf{u}^{\prime}(\varepsilon)\right)=\frac{1}{2} \partial_{3} u_{\alpha}(\varepsilon) \rightarrow 0$ strongly in $C\left([0, T] ; L^{2}(\Omega)\right)$. Therefore we have proved (5.17) and then (5.16) follows by the Korn inequality. Thus we have proved the strong convergence of displacements

$$
\mathbf{u}(\varepsilon) \rightarrow \mathbf{u} \quad \text { strongly in } C\left([0, T] ; H^{1}(\Omega) \times H^{1}(\Omega) \times L^{2}(\Omega)\right) .
$$

\section{A Spherical surface}

Let $\omega=\left[0, d_{1}\right] \times\left[0, d_{2}\right]$, where $d_{1} \in(0,2 \pi], d_{2} \in(0, \pi]$, with one of the strict inequalities $d_{1}<2 \pi$ or $d_{2}<\pi$ holding, and let $(\varphi, \theta)$ denotes the generic point in $\omega$. Let $R>0$. We define a spherical shell by the parametrization

$$
\mathbf{X}: \omega \rightarrow \mathbb{R}^{3}, \quad \mathbf{X}(\varphi, \theta)=(R \sin \theta \cos \varphi, R \sin \theta \sin \varphi, R \cos \theta)^{T} .
$$


Then the extended covariant basis of the shell $S=\mathbf{X}(\omega)$ is given by

$$
\begin{aligned}
& \mathbf{a}_{1}(\varphi, \theta)=\partial_{\varphi} \mathbf{X}(\varphi, \theta)=R(-\sin \theta \sin \varphi, \sin \theta \cos \varphi, 0)^{T}, \\
& \mathbf{a}_{2}(\varphi, \theta)=\partial_{\theta} \mathbf{X}(\varphi, \theta)=R(\cos \theta \cos \varphi, \cos \theta \sin \varphi,-\sin \theta)^{T}, \\
& \mathbf{a}_{3}(\varphi, \theta)=\frac{\mathbf{a}_{1}(\varphi, \theta) \times \mathbf{a}_{2}(\varphi, \theta)}{\left|\mathbf{a}_{1}(\varphi, \theta) \times \mathbf{a}_{2}(\varphi, \theta)\right|}=(-\sin \theta \cos \varphi,-\sin \theta \sin \varphi,-\cos \theta)^{T} .
\end{aligned}
$$

The contravariant basis is biorthogonal and is given by

$$
\begin{aligned}
\mathbf{a}^{1}(\varphi, \theta) & =\frac{1}{R}(-\sin \varphi / \sin \theta, \cos \varphi / \sin \theta, 0)^{T}, \\
\mathbf{a}^{2}(\varphi, \theta) & =\frac{1}{R}(\cos \theta \cos \varphi, \cos \theta \sin \varphi,-\sin \theta)^{T}, \\
\mathbf{a}^{3}(\varphi, \theta) & =(-\sin \theta \cos \varphi,-\sin \theta \sin \varphi,-\cos \theta)^{T} .
\end{aligned}
$$

The covariant $\mathbf{A}_{c}=\left(a_{\alpha \beta}\right)$ and contravariant $\mathbf{A}^{c}=\left(a^{\alpha \beta}\right)$ metric tensors are respectively given by

$$
\mathbf{A}_{c}=\left[\begin{array}{cc}
R^{2} \sin ^{2} \theta & 0 \\
0 & R^{2}
\end{array}\right], \quad \mathbf{A}^{c}=\left[\begin{array}{cc}
\frac{1}{R^{2}} \frac{1}{\sin ^{2} \theta} & 0 \\
0 & \frac{1}{R^{2}}
\end{array}\right]
$$

and the area element is now $\sqrt{a} d S=\sqrt{\operatorname{det} \mathbf{A}_{c}} d S=R^{2} \sin \theta d S$. The covariant and mixed components of the curvature tensor are now given by

$$
\begin{aligned}
b_{11} & =\mathbf{a}^{3} \cdot \partial_{\varphi} \mathbf{a}_{1}=R \sin ^{2} \theta, \quad b_{12}=\mathbf{a}^{3} \cdot \partial_{\theta} \mathbf{a}_{1}=0, \\
b_{21} & =\mathbf{a}^{3} \cdot \partial_{\varphi} \mathbf{a}_{2}=0, \quad b_{22}=\mathbf{a}^{3} \cdot \partial_{\theta} \mathbf{a}_{2}=R, \\
b_{1}^{1} & =\sum_{\sigma=1}^{2} a^{1 \sigma} b_{\sigma 1}=a^{11} b_{11}=\frac{1}{R}, \quad b_{2}^{1}=\sum_{\sigma=1}^{2} a^{1 \sigma} b_{\sigma 2}=0, \\
b_{1}^{2} & =\sum_{\sigma=1}^{2} a^{2 \sigma} b_{\sigma 1}=0, \quad b_{2}^{2}=\sum_{\sigma=1}^{2} a^{2 \sigma} b_{\sigma 2}=a^{22} b_{22}=\frac{1}{R^{2}} R=\frac{1}{R} .
\end{aligned}
$$

For Christoffel symbols $\Gamma_{\alpha \beta}^{\sigma}=\mathbf{a}^{\sigma} \cdot \partial_{\beta} \mathbf{a}_{\alpha}$ one has

$$
\Gamma^{1}=\left(\Gamma_{\alpha \beta}^{1}\right)=\left[\begin{array}{cc}
0 & \operatorname{ctg} \theta \\
\operatorname{ctg} \theta & 0
\end{array}\right], \quad \Gamma^{2}=\left(\Gamma_{\alpha \beta}^{2}\right)=\left[\begin{array}{cc}
-\sin \theta \cos \theta & 0 \\
0 & 0
\end{array}\right] .
$$

Now the displacement vector $\tilde{\mathbf{v}}$ in the canonical coordinates is rewritten in the local basis $\tilde{\mathbf{v}}=$ $\mathbf{Q v}=v_{1} \mathbf{a}^{1}+v_{2} \mathbf{a}^{2}+v_{3} \mathbf{a}^{3}$. Note that contravariant basis is different than the usual basis associated with the spherical coordinates. One has

$$
v_{1}=R \sin \theta v_{\varphi}, \quad v_{2}=R v_{\theta}, \quad v_{3}=-v_{r} .
$$

Similarly, $\tilde{\mathcal{P}}_{ \pm}=\mathbf{Q}^{-T} \mathcal{P}_{ \pm}=\left(\mathcal{P}_{ \pm}\right)_{1} \mathbf{a}_{1}+\left(\mathcal{P}_{ \pm}\right)_{2} \mathbf{a}_{2}+\left(\mathcal{P}_{ \pm}\right)_{3} \mathbf{a}_{3}$. Thus

$$
\left(\mathcal{P}_{ \pm}\right)_{1}=\frac{1}{R \sin \theta}\left(\mathcal{P}_{ \pm}\right)_{\varphi}, \quad\left(\mathcal{P}_{ \pm}\right)_{2}=\frac{1}{R}\left(\mathcal{P}_{ \pm}\right)_{\theta}, \quad\left(\mathcal{P}_{ \pm}\right)_{3}=-\left(\mathcal{P}_{ \pm}\right)_{r}
$$

and

$$
\mathcal{P}_{ \pm} \cdot \mathbf{v}=\left(\mathcal{P}_{ \pm}\right)_{1} v_{1}+\left(\mathcal{P}_{ \pm}\right)_{2} v_{2}+\left(\mathcal{P}_{ \pm}\right)_{3} v_{3}=\left(\mathcal{P}_{ \pm}\right)_{\varphi} v_{\varphi}+\left(\mathcal{P}_{ \pm}\right)_{\theta} v_{\theta}+\left(\mathcal{P}_{ \pm}\right)_{r} v_{r}
$$

Inserting the geometry coefficients into the strain $\gamma$ we obtain

$$
\begin{aligned}
\gamma(\mathbf{v}) & =\left[\begin{array}{cc}
\partial_{1} v_{1}-\sum_{\kappa=1}^{2} \Gamma_{11}^{\kappa} v_{\kappa}-b_{11} v_{3} & \frac{1}{2}\left(\partial_{1} v_{2}+\partial_{2} v_{1}\right)-\sum_{\kappa=1}^{2} \Gamma_{12}^{\kappa} v_{\kappa}-b_{12} v_{3} \\
\frac{1}{2}\left(\partial_{2} v_{1}+\partial_{1} v_{2}\right)-\sum_{\kappa=1}^{2} \Gamma_{21}^{\kappa} v_{\kappa}-b_{21} v_{3} & \partial_{2} v_{2}-\sum_{\kappa=1}^{2} \Gamma_{22}^{\kappa} v_{\kappa}-b_{22} v_{3}
\end{array}\right] \\
& =R\left[\begin{array}{cc}
\sin \theta \partial_{\varphi} v_{\varphi}+\sin \theta \cos \theta v_{\theta}+\sin ^{2} \theta v_{r} & \frac{1}{2}\left(\partial_{\varphi} v_{\theta}+\sin \theta \partial_{\theta} v_{\varphi}\right)-\cos \theta v_{\varphi} \\
\frac{1}{2}\left(\sin \theta \partial_{\theta} v_{\varphi}+\partial_{\varphi} v_{\theta}\right)-\cos \theta v_{\varphi} & \partial_{\theta} v_{\theta}+v_{r}
\end{array}\right] .
\end{aligned}
$$


Next

$$
\begin{aligned}
\tilde{\mathcal{C}}\left(\mathbf{A}^{c} \boldsymbol{\gamma}(\mathbf{u})\right): \gamma(\mathbf{v}) \mathbf{A}^{c}=\frac{2 \tilde{\lambda}}{\tilde{\lambda}+2} \operatorname{tr}\left(\mathbf{A}^{c} \boldsymbol{\gamma}(\mathbf{u})\right) \operatorname{tr}\left(\mathbf{A}^{c} \boldsymbol{\gamma}(\mathbf{v})\right)+2 \mathbf{A}^{c} \boldsymbol{\gamma}(\mathbf{u}): \boldsymbol{\gamma}(\mathbf{v}) \mathbf{A}^{c} \\
=\frac{2 \tilde{\lambda}}{\tilde{\lambda}+2} \frac{1}{R^{2}}\left(\frac{1}{\sin ^{2} \theta}\left(\sin \theta \partial_{\varphi} u_{\varphi}+\sin \theta \cos \theta u_{\theta}+\sin ^{2} \theta u_{r}\right)+\left(\partial_{\theta} u_{\theta}+u_{r}\right)\right) \\
\left(\frac{1}{\sin ^{2} \theta}\left(\sin \theta \partial_{\varphi} v_{\varphi}+\sin \theta \cos \theta v_{\theta}+\sin ^{2} \theta v_{r}\right)+\left(\partial_{\theta} v_{\theta}+v_{r}\right)\right) \\
\quad+2 \frac{1}{R^{2}}\left(\frac{1}{\sin ^{4} \theta}\left(\sin \theta \partial_{\varphi} u_{\varphi}+\sin \theta \cos \theta u_{\theta}+\sin ^{2} \theta u_{r}\right)\left(\sin \theta \partial_{\varphi} v_{\varphi}+\sin \theta \cos \theta v_{\theta}+\sin ^{2} \theta v_{r}\right)\right. \\
\quad+\frac{2}{\sin ^{2} \theta}\left(\frac{1}{2}\left(\partial_{\varphi} u_{\theta}+\sin \theta \partial_{\theta} u_{\varphi}\right)-\cos \theta u_{\varphi}\right)\left(\frac{1}{2}\left(\partial_{\varphi} v_{\theta}+\sin \theta \partial_{\theta} v_{\varphi}\right)-\cos \theta v_{\varphi}\right) \\
\left.\quad+\left(\partial_{\theta} u_{\theta}+u_{r}\right)\left(\partial_{\theta} v_{\theta}+v_{r}\right)\right) \\
=\frac{2 \tilde{\lambda}}{\tilde{\lambda}+2} \frac{1}{R^{2}}\left(\frac{1}{\sin \theta}\left(\partial_{\varphi} u_{\varphi}+\cos \theta u_{\theta}+\sin \theta u_{r}\right)+\partial_{\theta} u_{\theta}+u_{r}\right) \\
\left(\frac{1}{\sin \theta}\left(\partial_{\varphi} v_{\varphi}+\cos \theta v_{\theta}+\sin \theta v_{r}\right)+\partial_{\theta} v_{\theta}+v_{r}\right) \\
\quad+2 \frac{1}{R^{2}}\left(\frac{1}{\sin ^{2} \theta}\left(\partial_{\varphi} u_{\varphi}+\cos \theta u_{\theta}+\sin \theta u_{r}\right)\left(\partial_{\varphi} v_{\varphi}+\cos \theta v_{\theta}+\sin \theta v_{r}\right)\right. \\
\left.\quad+\frac{1}{2 \sin ^{2} \theta}\left(\partial_{\varphi} u_{\theta}+\sin \theta \partial_{\theta} u_{\varphi}-2 \cos \theta u_{\varphi}\right)\left(\partial_{\varphi} v_{\theta}+\sin \theta \partial_{\theta} v_{\varphi}-2 \cos \theta v_{\varphi}\right)+\left(\partial_{\theta} u_{\theta}+u_{r}\right)\left(\partial_{\theta} v_{\theta}+v_{r}\right)\right)
\end{aligned}
$$

The insertion of the above expression for $\tilde{\mathcal{C}}$ into (3.23) gives the equations of the spherical membrane shell. They read: find $\left\{\left(u_{\varphi}, u_{\theta}, u_{r}\right)\right\} \in C\left([0, T] ; \mathcal{V}_{M}(\omega)\right)$, satisfying the system

$$
\begin{gathered}
\int_{0}^{d_{1}} \int_{0}^{d_{2}}\left(\left(\frac{2 \tilde{\lambda}}{\tilde{\lambda}+2}+\frac{4 \alpha^{2}}{(\tilde{\lambda}+2)\left(\beta(\tilde{\lambda}+2)+\alpha^{2}\right)}\right) \frac{1}{R^{2}}\left(\frac{1}{\sin \theta}\left(\partial_{\varphi} u_{\varphi}+\cos \theta u_{\theta}+\sin \theta u_{r}\right)+\partial_{\theta} u_{\theta}+u_{r}\right)\right. \\
\left(\frac{1}{\sin \theta}\left(\partial_{\varphi} v_{\varphi}+\cos \theta v_{\theta}+\sin \theta v_{r}\right)+\partial_{\theta} v_{\theta}+v_{r}\right) \\
\quad+2 \frac{1}{R^{2}}\left(\frac{1}{\sin ^{2} \theta}\left(\partial_{\varphi} u_{\varphi}+\cos \theta u_{\theta}+\sin \theta u_{r}\right)\left(\partial_{\varphi} v_{\varphi}+\cos \theta v_{\theta}+\sin \theta v_{r}\right)\right. \\
\quad+\frac{1}{2 \sin ^{2} \theta}\left(\partial_{\varphi} u_{\theta}+\sin \theta \partial_{\theta} u_{\varphi}-2 \cos \theta u_{\varphi}\right)\left(\partial_{\varphi} v_{\theta}+\sin \theta \partial_{\theta} v_{\varphi}-2 \cos \theta v_{\varphi}\right) \\
\left.\left.+\left(\partial_{\theta} u_{\theta}+u_{r}\right)\left(\partial_{\theta} v_{\theta}+v_{r}\right)\right)\right) R^{2} \sin \theta d \varphi d \theta \\
=\int_{0}^{d_{1}} \int_{0}^{d_{2}}\left(\left(\left(\mathcal{P}_{+}\right)_{\varphi}+\left(\mathcal{P}_{-}\right)_{\varphi}\right) v_{\varphi}+\left(\left(\mathcal{P}_{+}\right)_{\theta}+\left(\mathcal{P}_{-}\right)_{\theta}\right) v_{\theta}+\left(\left(\mathcal{P}_{+}\right)_{r}+\left(\mathcal{P}_{-}\right)_{r}\right) v_{r}\right) R^{2} \sin \theta d \varphi d \theta \\
\left(v_{\varphi}, v_{\theta}, v_{r}\right) \in \mathcal{V}_{M}(\omega) .
\end{gathered}
$$

Then $\int_{-1 / 2}^{1 / 2} p^{0} d r$ is calculated from (3.22) and the fluctuation of the pressure across the thickness can be calculated from (3.25).

Remark 20. In the case of the whole sphere we are not in the elliptic membrane case since the boundary conditions are different. However if we assume that the body is loaded radially (i.e. $\mathcal{P}_{ \pm}, V$ are functions of time only) we can search for the radial solution of the three-dimensional problem (3.16) (i.e. $u_{\varphi}=u_{\theta}=0$ and $u_{r}$ is independent of $\varphi$ and $\theta$ ). The asymptotic analysis is based on the 
a priori estimates used in Theorem 12. In the case of the whole sphere and radial solutions, a simple calculation gives

$$
\begin{aligned}
\left\|\gamma^{\varepsilon}(\mathbf{v})\right\|_{L^{2}\left(\Omega ; \mathbb{R}^{3 \times 3}\right)}^{2} & =\int_{0}^{2 \pi} \int_{0}^{\pi} \int_{-1 / 2}^{1 / 2}\left(\left(R \sin ^{2} \theta-\varepsilon z_{3}\right)^{4} v_{3}^{2}+\left(R-\varepsilon z_{3}\right)^{2} v_{3}^{2}+\frac{1}{\varepsilon^{2}} \partial_{3} v_{3}\left(z_{3}\right)^{2}\right) d z_{3} d \theta d \varphi \\
& \geq \int_{0}^{2 \pi} \int_{0}^{\pi} \int_{-1 / 2}^{1 / 2}\left(R^{2} / 4 v_{3}^{2}+\frac{1}{\varepsilon^{2}} \partial_{3} v_{3}\left(z_{3}\right)^{2}\right) d z_{3} d \theta d \varphi \geq C^{2}\left\|v_{3}\right\|_{H^{1}(\Omega)}^{2},
\end{aligned}
$$

for $R \geq \varepsilon$ and $1 \gg \varepsilon$. Then the subsequent analysis follows as above and the limit model in this case can be obtained by specialization of the above equations for spherical geometry.

Thus for loading depending only on time for the solution of the shell problem we obtain the following relation for $u_{r}$ and $p^{0}$ :

$$
\begin{aligned}
& \frac{3 \tilde{\lambda}+2}{\tilde{\lambda}+2} 4 u_{r}-2 R \frac{2 \alpha}{\tilde{\lambda}+2} \int_{-1 / 2}^{1 / 2} p^{0} d r=\left(\left(\mathcal{P}_{+}\right)_{r}+\left(\mathcal{P}_{-}\right)_{r}\right) R^{2} . \\
& \frac{d}{d t} \int_{-1 / 2}^{1 / 2}\left(\beta+\frac{\alpha^{2}}{\tilde{\lambda}+2}\right) p^{0} q R^{2} d r+2 R \frac{2 \alpha}{\tilde{\lambda}+2} \int_{-1 / 2}^{1 / 2} \partial_{t} u_{r} q d r+R^{2} \int_{-1 / 2}^{1 / 2} \frac{\partial p^{0}}{\partial r} \frac{\partial q}{\partial r} d r= \\
& V(q(-1 / 2)-q(1 / 2)) R^{2} \quad \text { in } \quad \mathcal{D}^{\prime}(0, T), \quad q \in H^{1}(-1 / 2,1 / 2), \\
& p^{0}=0 \quad \text { at } t=0 .
\end{aligned}
$$

From (A.22) for constant test functions we obtain

$$
\left(\beta+\frac{\alpha^{2}}{\tilde{\lambda}+2}\right) \partial_{t} \int_{-1 / 2}^{1 / 2} p^{0} R^{2} d r+2 R \frac{2 \alpha}{\tilde{\lambda}+2} \partial_{t} u_{r}=0
$$

Inserting $u_{r}$ from(A.21), after some calculations, we obtain

$$
\partial_{t} \int_{-1 / 2}^{1 / 2} p^{0}=-\frac{R \alpha}{\beta(3 \tilde{\lambda}+2)+\alpha^{2} \frac{3 \tilde{\lambda}+6}{\tilde{\lambda}+2}} \partial_{t}\left(\left(\mathcal{P}_{+}\right)_{r}+\left(\mathcal{P}_{-}\right)_{r}\right) .
$$

After partial integration in (A.22), we obtain

$$
\begin{aligned}
& \left(\beta+\frac{\alpha^{2}}{\tilde{\lambda}+2}\right) \partial_{t} p^{0} R^{2}+2 R \frac{2 \alpha}{\tilde{\lambda}+2} \partial_{t} u_{r}-R^{2} \partial_{r r} p^{0}=0, \\
& \left.\partial_{r} p^{0}\right|_{r= \pm 1 / 2}=V \\
& p^{0}=0 \quad \text { at } \quad t=0 .
\end{aligned}
$$

Here we calculate $u_{r}$ from (A.21) with $\partial_{t} \int_{-1 / 2}^{1 / 2} p^{0} d r$ replaced using (A.23) and obtain the boundary value problem for $p^{0}$

$$
\begin{aligned}
& \left(\beta+\frac{\alpha^{2}}{\tilde{\lambda}+2}\right) \partial_{t} p^{0}+R \frac{\alpha\left(\beta(\tilde{\lambda}+2)+\alpha^{2}\right)}{\beta(3 \tilde{\lambda}+2)(\tilde{\lambda}+2)+\alpha^{2}(3 \tilde{\lambda}+6)} \partial_{t}\left(\left(\mathcal{P}_{+}\right)_{r}+\left(\mathcal{P}_{-}\right)_{r}\right)-\partial_{r r} p^{0}=0, \\
& \left.\partial_{r} p^{0}\right|_{r= \pm 1 / 2}=V \\
& p^{0}=0 \quad \text { at } \quad t=0 .
\end{aligned}
$$

This equation has the same structure as the equation in [31] for spherical poroelastic membrane, however the coefficients are obviously not the same since in [31] there are no inverse Biot's coefficient $\beta$ and the effective stress coefficient $\alpha$. In the constitutive law (2.6) Taber takes $\alpha=1$ and in (2.8) $\beta$ seems to be 1 as well. Hence, our consideration establish rigorously the results from [31]. 


\section{B Acknowledgements}

The research of A.M. was supported in part by the LABEX MILYON (ANR-10-LABX-0070) of Université de Lyon, within the program "Investissements d'Avenir" (ANR-11-IDEX-0007) operated by the French National Research Agency (ANR).

\section{References}

[1] J. Blaauwendraad, J.H. Hoefakker, Structural Shell Analysis: Understanding and Application, Springer, Dordrecht, 2014.

[2] D. Blanchard, G. Francfort, Asymptotic thermoelastic behavior of flat plates, Quarterly of Applied Mathematics 45 (1987), 645-667.

[3] G. Cederbaum, L. Li, K. Schulgasser, (Eds.), Poroelastic structures. Elsevier, (2000).

[4] P. G. Ciarlet, An introduction to differential geometry with applications to elasticity, Springer, Dordrecht, 2005.

[5] P. G. Ciarlet, Mathematical elasticity. Vol. III. Theory of shells, North-Holland, Amsterdam 2000.

[6] P.G. Ciarlet and P. Destuynder, A justification of the two dimensional linear plate model, J. Mécanique 18 (1979), 315-344.

[7] P.G. Ciarlet and V. Lods, Asymptotic Analysis of Linearly Elastic Shells. I. Justification of Membrane Shells Equations, Arch. Rational Mech. Anal. 136 (1996), 119-161.

[8] P. G. Ciarlet, V. Lods and B. Miara, Asymptotic analysis of linearly elastic shells. II. Justification of flexural shell equations, Arch. Rational Mech. Anal. 136 (1996), 163-190.

[9] T. Clopeau, J.L. Ferrín, R. P. Gilbert and A. Mikelić, Homogenizing the Acoustic Properties of the Seabed, II, Mathematical and Computer Modelling 33 (2001), 821-841.

[10] O. Coussy, Mechanics and Physics of Porous Solids, John Wiley and Sons, Chichester, 2011.

[11] S. C. Cowin, Bone poroelasticity, Journal of Biomechanics, 32 (1999), 217-238.

[12] M. Dauge, E. Faou and Z. Yosibash, Plates and shells: Asymptotic expansions and hierarchical models, in "Encyclopedia of Computational Mechanics", edited by E. Stein, R. de Borst and T. J.R. Hughes, John Wiley and Sons, Ltd., 2004, 199-236.

[13] A. Di Carlo, V. Varano, V. Sansalone, A. Tatone, Living shell-like structures, Applied and Industrial Mathematics In Italy-II, 75 (2007), 315-326.

[14] G. Duvaut, J. L. Lions, Inequalities in Mechanics and Physics, volume 219 of Grundlehren der mathematischen Wissenschaften, Springer, Heidelberg 1976.

[15] M. Etchessahar, S. Sahraoui, B. Brouard, Bending vibrations of a rectangular poroelastic plate, C. R. Acad. Sci. Paris, Série II b, 329 (2001), 615620.

[16] J.L. Ferrín and A. Mikelić, Homogenizing the Acoustic Properties of a Porous Matrix Containing an Incompressible Inviscid Fluid, Mathematical Methods in the Applied Sciences 26 (2003), 831-859. 
[17] N. Grosjean, D. Iliev, O. Iliev, R. Kirsch, Z. Lakdawala, M. Lance, M. Michard, A. Mikelić, Experimental and numerical study of the interaction between fluid flow and filtering media on the macroscopic scale, Separation and Purification Technology, 156, Part 1 (2015), p. 22-27.

[18] J.H. Hoefakker, Theory Review for Cylindrical Shells and Parametric Study of Chimneys and Tanks, PhD thesis, Delft University of Technology, 2010.

[19] O.P. Iliev, A.E. Kolesov, P.N. Vabishchevich, Numerical Solution of Plate Poroelasticity Problems, Transp Porous Med., 115 (2016), 563-580.

[20] A. Marciniak-Czochra and A. Mikelić, A Rigorous Derivation of the Equations for the Clamped Biot-Kirchhoff-Love Poroelastic plate, Arch. Rational Mech. Anal. 215 (2015), 1035-1062.

[21] C. C. Mei and B. Vernescu, Homogenization Methods for Multiscale Mechanics, World Scientific Publishing Co., 2010.

[22] A. Mikelić and J. Tambača, Derivation of a poroelastic flexural shell model, Multiscale Model. Simul. 14 (2016), 364-397.

[23] A. Mikelić and M. F. Wheeler, On the interface law between a deformable porous medium containing a viscous fluid and an elastic body, Mathematical Models and Methods in Applied Sciences, 22 (2012), 11, 1240031 (32 pages).

[24] A. Mikelić, M. F. Wheeler, Theory of the dynamic Biot-Allard equations and their link to the quasi-static Biot system, Journal of Mathematical Physics, 53, (2012), 123702 (15 pages).

[25] P.M. Naghdi, The Theory of Shells and Plates, Encyclopedia of Physics Vol. VIa/2, Springer, New York, 1972, pp. 423-640.

[26] Nguetseng G, Asymptotic analysis for a stiff variational problem arising in mechanics, SIAM J. Math. Anal. 20 (1990), 608-623.

[27] J.L. Nowinski, C.F. Davis, A model of the human skull as a poroelastic spherical shell subjected to a quasistatic load, Mathematical Biosciences, 8 (1970), 397-416.

[28] E. Sanchez-Palencia, Non-Homogeneous Media and Vibration Theory, Springer Lecture Notes in Physics 129, Springer, 1980.

[29] J. Simon, Compact sets in the space $L^{p}(0, T ; B)$, Annali di Matematica Pura ed Applicata, 146 (1986), 65-96.

[30] L. A. Taber, A Theory for Transverse Deflection of Poroelastic Plates, J. Appl. Mech., Vol. 59 (1992), p. 628-634.

[31] L. A. Taber and A. M. Puleo, Poroelastic Plate and Shell Theories, in A. P. S. Selvadurai (ed.), Mechanics of Poroelastic Media, Kluwer Academic Publishers 1996, 323-337.

[32] D. D. Theodorakopoulos, D. E. Beskos, Flexural vibrations of poroelastie plates, Acta Mechanica, 103 (1994), 191-203.

[33] I. Tolstoy, ed., Acoustics, elasticity, and thermodynamics of porous media. Twenty-one papers by M.A. Biot, Acoustical Society of America, New York, 1992.

[34] L. Yeghiazarian, K. Pillai, R. Rosati (eds), Special Issue: Thin Porous Media, Transp Porous Med., 115, Issue 3, December 2016. 